ARTICLE

\title{
The IncRNA lincNMR regulates nucleotide metabolism via a YBX1 - RRM2 axis in cancer
}

Minakshi Gandhi (10) 1,2,8, Matthias Groß', Jessica M. Holler ${ }^{3}$, Si'Ana A. Coggins ${ }^{3}$, Nitin Patil ${ }^{4}$, Joerg H. Leupold ${ }^{4}$, Mathias Munschauer (1D ${ }^{5}$, Monica Schenone (10 ${ }^{5}$, Christina R. Hartigan ${ }^{5}$, Heike Allgayer (10 ${ }^{4}$, Baek Kim (D) ${ }^{3,6} \&$ Sven Diederichs (10) $1,7 \times$

Long intergenic non-coding RNA-Nucleotide Metabolism Regulator (lincNMR) is a long noncoding RNA (IncRNA) which is induced in hepatocellular carcinoma. Its depletion invokes a proliferation defect, triggers senescence and inhibits colony formation in liver, but also breast and lung cancer cells. Triple-label SILAC proteomics profiles reveal a deregulation of key cell cycle regulators in lincNMR-depleted cells like the key dNTP synthesizing enzymes RRM2, TYMS and TK1, implicating lincNMR in regulating nucleotide metabolism. LincNMR silencing decreases dNTP levels, while exogenous dNTPs rescues the proliferation defect induced by lincNMR depletion. In vivo RNA Antisense Purification (RAP-MS) identifies YBX1 as a direct interaction partner of lincNMR which regulates RRM2, TYMS and TK1 expression and binds to their promoter regions. In a Chick Chorioallantoic Membrane (CAM) in vivo model, lincNMRdepleted tumors are significantly smaller. In summary, we discover a lincRNA, lincNMR, which regulates tumor cell proliferation through a YBX1-RRM2-TYMS-TK1 axis governing nucleotide metabolism.

\footnotetext{
${ }^{1}$ Division of RNA Biology \& Cancer, German Cancer Research Center (DKFZ), Heidelberg, Germany. ${ }^{2}$ German Academic Exchange Service (DAAD), Bonn, Germany; Helmholtz International Graduate School for Cancer Research (HIGS), Heidelberg, Germany; Faculty of Biosciences, Heidelberg University, Heidelberg, Germany. ${ }^{3}$ Department of Pediatrics, School of Medicine, Emory University, Atlanta, GA, USA. ${ }^{4}$ Department of Experimental Surgery-Cancer Metastasis, Medical Faculty Mannheim, Centre for Biomedicine and Medical Technology Mannheim, University of Heidelberg, Mannheim, Germany. ${ }^{5}$ Broad Institute of MIT and Harvard, Cambridge, MA, USA. ${ }^{6}$ Center for Drug Discovery, Children's Healthcare of Atlanta, Atlanta, GA, USA. ${ }^{7}$ Division of Cancer Research, Department of Thoracic Surgery, Medical Center, Faculty of Medicine, University of Freiburg, German Cancer Consortium (DKTK)-Partner Site Freiburg, Freiburg, Germany. ${ }^{8}$ Present address: Cold Spring Harbor Laboratory, Cold Spring Harbor, NY, USA. ${ }^{凶}$ email: s.diederichs@dkfz.de
} 
comprehensive landscape of the transcriptome has revealed that although over a $75 \%$ of human genome is transcribed, only about $2 \%$ of it encodes for proteins ${ }^{1}$. In recent years, over 10,000 long non-coding RNAs (lncRNAs) have been identified, and their number is ever increasing. Although lncRNAs were initially viewed as background noise from junk DNA, it is now evident that they play an important role in various biological processes, and their deregulation has been linked to various cancers including liver cancer ${ }^{2,3}$. LncRNAs act as drivers of carcinogenesis by regulating one or multiple hallmarks of cancer ${ }^{4-6}$. Several well-studied examples include lncRNAs regulating viability ${ }^{7-9}$, proliferation ${ }^{10-12}$, growth suppression ${ }^{13,14}$, migration $^{15-17}$, angiogenesis ${ }^{18,19}$, and cellular immortality ${ }^{20-22}$. Detailed epigenetic, genomic, and transcriptional analyses have revealed that lncRNAs have cancer type-specific deregulation patterns ${ }^{23}$ and could be attractive molecules for therapeutic applications ${ }^{24}$. LncRNAs are heterogeneous molecules and play diverse functional roles by interacting with DNA, RNA, or proteins, such as recruitment of chromatin modifying complexes ${ }^{25}$ or transcription factors ${ }^{26}$, controlling mRNA stability ${ }^{27}$, and acting as competing endogenous RNAs ${ }^{28}$.

Liver cancer is the fourth leading cause of cancer-related mortalities worldwide in 2018 , and is predicted to be sixth most commonly diagnosed cancer ${ }^{29}$. Of all liver cancers diagnosed, primary liver cancer called hepatocellular carcinoma (HCC) constitutes $75-85 \%$ of the cases with limited treatment options in advanced stages warranting further investigations. Notably, lncRNAs like HULC, TERC, and HOTAIR have also been implicated in hepatocarcinogenesis $28,30,31$

In this study, we investigate lncRNAs induced in liver cancer patient samples derived from high-throughput RNA sequencing data and identify the lncRNA lincNMR (long intergenic noncoding RNA-nucleotide metabolism regulator) upregulated in HCC and impacting tumor growth in vivo. Its in-depth molecular characterization unravels its role in controlling nucleotide metabolism via interacting with YBX1 and regulating RRM2, TK1, and TYMS

\section{Results}

lincNMR is upregulated in hepatocellular carcinoma. To identify long noncoding RNAs (lncRNAs) deregulated in hepatocellular carcinoma (HCC), lncRNA expression was analyzed genome-wide based on the TCGA RNA sequencing dataset of liver cancer patients (tumor $=200$ samples, normal $=50$ samples). Out of 12,727 annotated lncRNAs in the TANRIC liver cancer dataset $^{32}, 217$ lncRNAs were found to be significantly $(P<0.05)$ induced by at least fivefold (median tumor/normal). In total, 49 lncRNAs were selected based on their genomic location, repeat content, pseudogene content, and association with clinical properties, and their expression was validated in nine liver cancer cell lines (Supplementary Data 1). Nine expressed lncRNAs were further selected based on expression levels, coding potential, and novelty for RNAi-based phenotypic analysis using siPOOLs ${ }^{33}$. Among these candidates, the uncharacterized transcript RP6$65 \mathrm{G} 23.3$ yielded the strongest decrease of cell viability as determined by ATP content measurement in liver cancer cells, comparable with the effect of $P L K 1^{34}$ or $H U L C^{35}$ knockdown (Fig. 1a). For reasons described below, we named this lncRNA lincNMR (long intergenic noncoding RNA-nucleotide metabolism regulator).

Basic characterization of lincNMR. LincNMR is a lncRNA transcribed from a bidirectional promoter in a head-to-head orientation on chromosome 14. Since the lincNMR transcript had never been studied, we defined its gene boundaries using rapid amplification of cDNA ends (RACE). 5'RACE identified a transcription start site (TSS) upstream of the current GENCODE annotation (Supplementary Fig. 1a). This finding was supported by RNA-Pol II Chip and switchgear TSS datasets (Supplementary Fig. 1b) corroborating the extended transcript identified in our $5^{\prime}$ RACE. $3^{\prime}$-RACE confirmed the previously annotated $3^{\prime}$-end of lincNMR, but also identified a second, less abundant isoform of lincNMR, including an additional internal exon (Supplementary Fig. 1c). Both isoforms have been deposited into Genbank with the accession numbers MK652436 and MK652437, respectively. Our 5'- and 3'-RACE results were supported by ENCODE/Cold Spring Harbor long RNA-Seq tracks from the ENCODE consortium $^{36}$ (Supplementary Fig. 1a, c). Next, we analyzed the coding potential of lincNMR using scores from phyloCSF ${ }^{37}$ (Supplementary Fig. 1d) and the Coding Potential Calculator ${ }^{38}$ (Supplementary Fig. 1e). Both algorithms classified lincNMR as a noncoding transcript. We determined the copy number of lincNMR per cell with at least two to seven copies. Since the subcellular localization maybe linked to the biological function of a noncoding $\mathrm{RNA}^{39,40}$, we performed subcellular fractionation with fraction-specific controls NEAT1 (chromatin fraction), $R N U-1$ (nucleoplasmic fraction) and DANCR (cytoplasmic fraction). LincNMR predominantly localized with $60-70 \%$ to the cytoplasm, but also showed considerable abundance in the nucleoplasm (Supplementary Fig. 1f).

LincNMR depletion affects cell proliferation and induces senescence. To elucidate the cellular function of lincNMR, we depleted lincNMR using two independent siPOOLs for additional specificity and to exclude any off-target effects observed with single siRNAs ${ }^{33}$ in multiple cancer cell lines. Both siPOOLs knocked down lincNMR efficiently in multiple liver (Supplementary Fig. 2a), breast (Supplementary Fig. 2b), and lung (Supplementary Fig. 2c) cancer cell lines. Since lincNMR knockdown decreased cell viability in liver cancer cells (Fig. 1a), cell proliferation was determined by performing BrdU incorporation assays. LincNMR silencing with two independent siPOOLs resulted in $30-80 \%$ decrease in cell proliferation in four liver cancer cell lines (HLE, HLF, SNU-387, and FLC-4) (Fig. 1b). Depletion of lincNMR also impaired cell proliferation in three breast (MCF-7, KPL-1, and T47D) (Supplementary Fig. 2d) and three lung (A549, NCI-H460, and NCI-H1299) cancer cell lines (Supplementary Fig. 2e). The overexpression of lincNMR rescued the proliferation defect caused by lincNMR depletion attesting to its specificity (Fig. 1c). Furthermore, a cell cycle analysis using flow cytometry confirmed an increase of cells in the G0/G1 phase of the cell cycle after depletion of lincNMR in multiple cell lines (Figs. 1d and Supplementary S2f, g).

The arrest of cells in the G0 / G1 phase prompted us to evaluate the induction of senescence. Depletion of lincNMR triggered senescence in three liver cancer cells with two independent siPOOLs as evident by $\beta$-GAL-positive blue cells in SA- $\beta$-GAL assay (Fig. 1e, f). The induction of senescence was supported by the induction of the pro-inflammatory cytokines IL$1 \mathrm{a}$ and IL-1b, which are bona fide markers of the senescenceassociated secretary phenotype (SASP) as well as the senescenceassociated proteins EDN and IGFBP7 (Fig. 1g). The induction of senescence was largely independent of the expression of p53 or pRB (Supplementary Fig. 2h) and also did not have gross consistent effects on p53 or pRB signaling (Supplementary Fig. 2i). LincNMR knockdown neither had a consistent impact on apoptosis (Supplementary Fig. 3a) nor did its overexpression affect proliferation in cancer cells (Supplementary Fig. 3b).

lincNMR is induced in multiple cancer entities. In line with the impact of lincNMR knockdown in cell lines from different tumor 

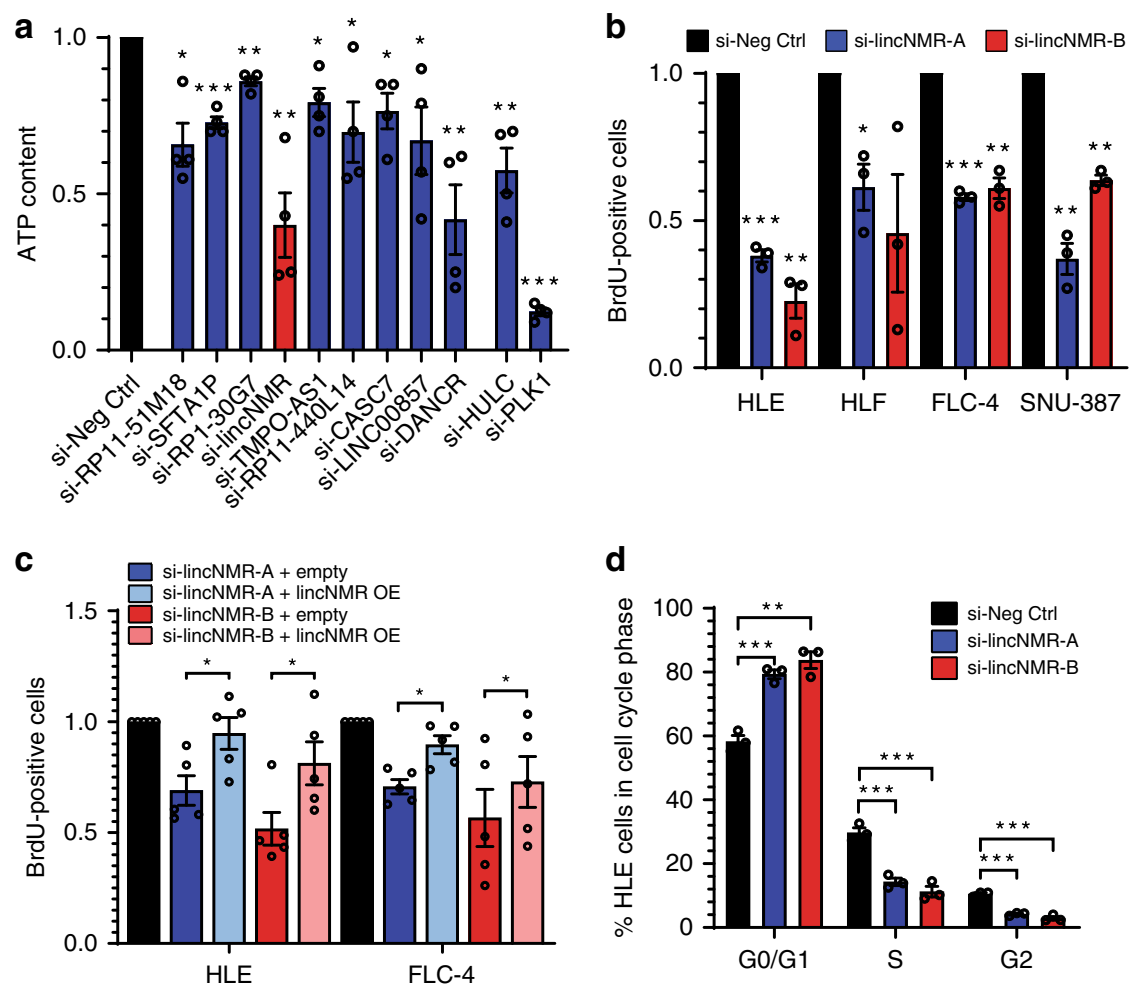

e
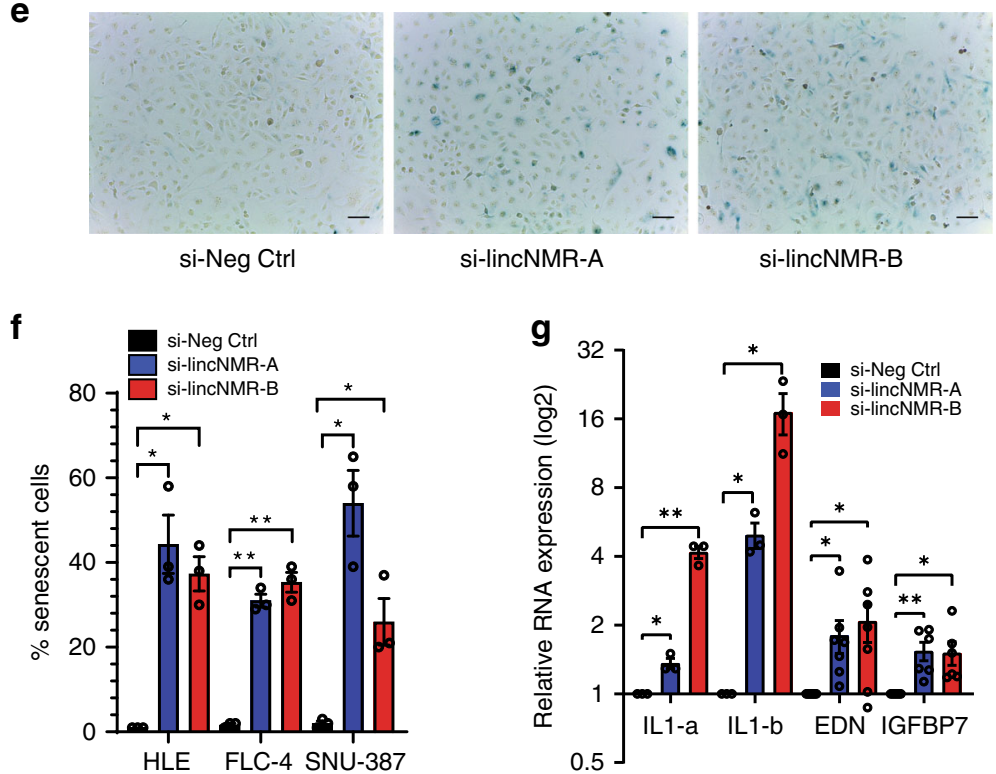

Fig. 1 LincNMR depletion impairs cell viability, cell proliferation and induces senescence. a Impact of depletion of selected IncRNAs with $10 \mathrm{nM}$ siPOOLs on cell viability as determined by CellTiter-Glo measuring the cellular ATP content after $72 \mathrm{~h}$ in HLE cells $(n=4)$. si-HULC and si-PLK1 served as positive controls. b Depletion of lincNMR with $10 \mathrm{nM}$ of two independent siPOOLs invokes a strong proliferation defect in four liver cancer cell lines (HLE, HLF, FLC4, and SNU-387) $72 \mathrm{~h}$ post transfection $(n=3)$. c Overexpression of lincNMR rescues the proliferation defect induced by lincNMR silencing in two different liver cancer cell lines, HLE and FLC-4. Data show BrdU assay readout at $72 \mathrm{~h}$ after lincNMR knockdown (KD), and $66 \mathrm{~h}$ after lincNMR overexpression (OE). Data shown are normalized to si-Neg Ctrl siPOOL transfected with empty vector pcDNA3.1 $(n=5)$. Significance was calculated by paired, two-tailed $t$ test with ${ }^{\star} P<0.05$. d Silencing of lincNMR with $10 \mathrm{nM}$ siPOOLs induces cell cycle arrest in the G0/G1 phase shown by flow cytometry $72 \mathrm{~h}$ post transfection in HLE cells $(n=3)$. Analysis was performed using FlowJo v10 software. e Representative microscopic images showing increased $\beta$-Gal activity indicating senescence in HLE cells $96 \mathrm{~h}$ post lincNMR knockdown with $10 \mathrm{nM}$ siPOOLs $(n=3)$. The scale bar represents $100 \mu \mathrm{m}$. f Bar graph representing percent $\beta$ Gal-positive cells in multiple liver cancer cell lines after lincNMR depletion $(n=3)$. $\mathbf{g}$ Induction of senescence-associated secretary phenotype (SASP) markers IL-1a, IL-1b, EDN, and IGFBP7 determined by RT-qPCR at $72 \mathrm{~h}$ after lincNMR knockdown in HLE cells with $10 \mathrm{nM}$ siPOOLs ( $n=3$ ). a-d, $\mathbf{f}, \mathbf{g}$ Data represent mean, and error bars represent SEM. a, b, d, f, g Significance was calculated by unpaired, two-tailed $t$ test with ${ }^{\star} P<0.05$; ${ }^{\star \star} P<0.01 ;{ }^{\star \star \star} P<0.001$. $\mathbf{a}-\mathbf{c}, \mathbf{g}$ Data shown were normalized to negative control siPOOL. 


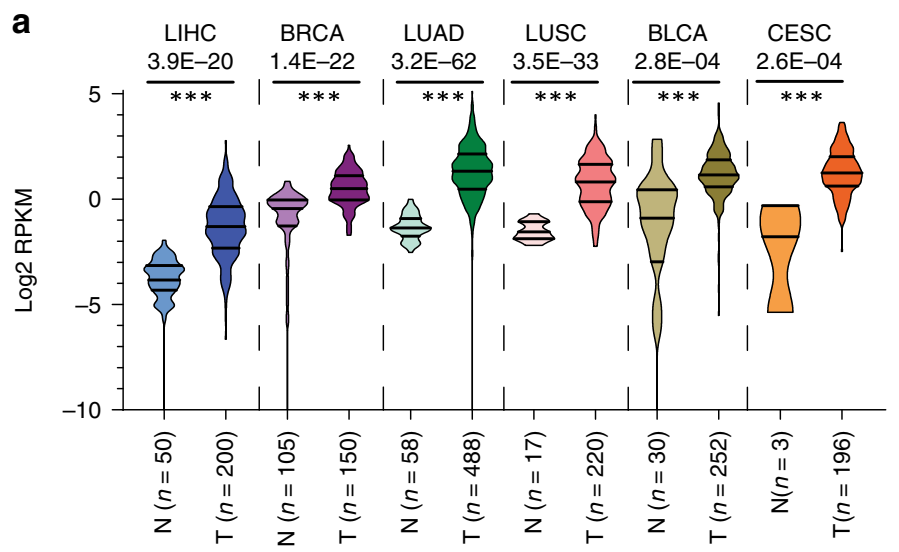

lincNMR expression \& regulation in multiple cancer types

b

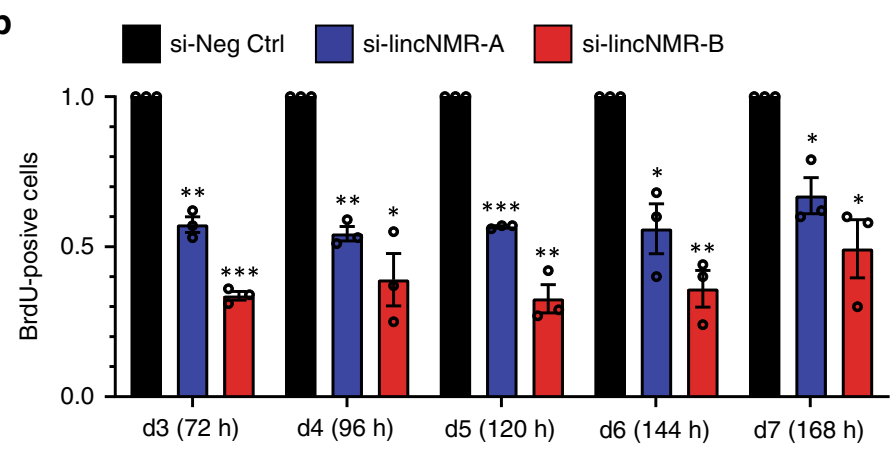

C

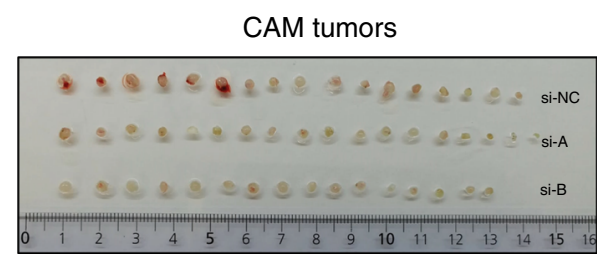

d

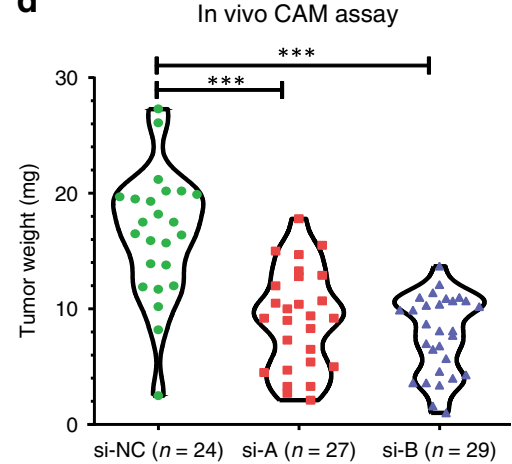

Fig. 2 lincNMR is induced in multiple cancer types and affects tumor growth in vivo. a LincNMR is expressed and regulated in multiple cancer types ( $N=$ normal, $T=$ tumor; data obtained from TCGA data portal). $\mathrm{LIHC}=$ hepatocellular carcinoma $(N=50, T=200), \mathrm{BRCA}=$ breast cancer $(N=105, T=105)$, LUAD = lung adenocarcinoma $(N=58, T=488), L U S C=$ lung squamous cell carcinoma $(N=17, T=220), B L C A=b l a d d e r$ cancer $(N=30, T=252)$, CESC $=$ cervical serous carcinoma $(N=3, T=196)$. Data are represented as log2 RPKM. b Timecourse of BrdU incorporation assays in HLE cells showing induction and maintenance of proliferation inhibition up to $168 \mathrm{~h}$ post lincNMR knockdown with $10 \mathrm{nM}$ siPOOLs $(n=3)$. Data are normalized to negative control siPOOL. Data represent mean, and error bars represent SEM. c Chick chorioallantoic membrane (CAM) assay: picture showing CAM-harvested tumors formed from HLE cells transfected with lincNMR or control siPOOLs. Tumors were harvested on day 6. LincNMR-depleted cells gave rise to smaller tumors (the number of chick embryos used in total: si-negative control $=24$, si-lincNMR-A $=27$, si-lincNMR-B $=29$ ). $\mathbf{d}$ Quantification of tumors harvested from CAM assay: decreased tumor weight in lincNMR-depleted tumors compared to the control group. $\mathbf{a}, \mathbf{b}$, $\mathbf{d}$ Significance was calculated by unpaired, twotailed $t$ test with ${ }^{\star} P<0.05 ;{ }^{\star \star} P<0.01 ;{ }^{\star \star \star} P<0.001$.

entities, lincNMR was also significantly induced between tumor and normal tissues across multiple cancer types, including breast invasive carcinoma, lung adenocarcinoma, lung squamous cell carcinoma, bladder urothelial carcinoma, and cervical squamous cell carcinoma (Fig. 2a). In addition, lincNMR was also expressed in a broad panel of 73 cell lines derived from different tumor entities or normal tissues (Supplementary Fig. 3c).

lincNMR depletion leads to decreased tumor growth in vivo. To test the impact of lincNMR knockdown on tumor growth in vivo, we turned to the chicken chorioallantoic membrane (CAM) model for in vivo xenograft experiments following the ethical responsibility aiming to replace, reduce or refine (3R) the use of animal models for research purposes. Knockdown efficiency (Supplementary Fig. 3d) and the presence of the growthinhibitory phenotype (Fig. 2b) were confirmed for the duration of the CAM assay until $168 \mathrm{~h}$ (day 7). After seeding lincNMRsilenced or control-treated cells onto the CAM, tumors were harvested, measured, and weighed. Tumors derived from lincNMR-depleted cells were significantly smaller in size than tumors derived from the control group (Fig. 2c). The tumor 


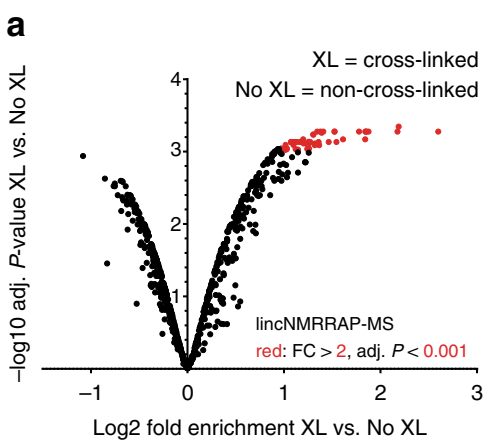

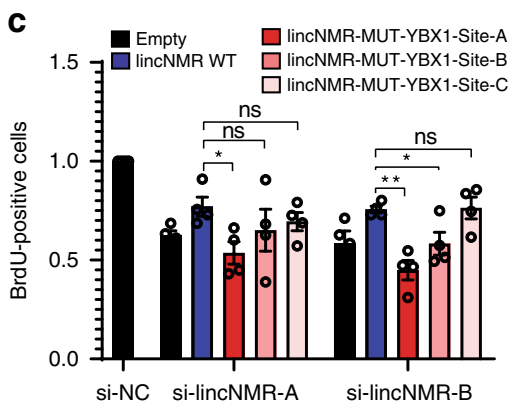

HCC survival: log10 log-rank $P$-value RAP-MS:log2 fold enrichment

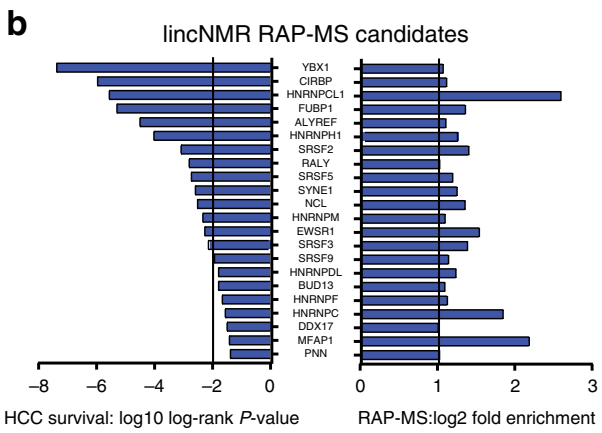

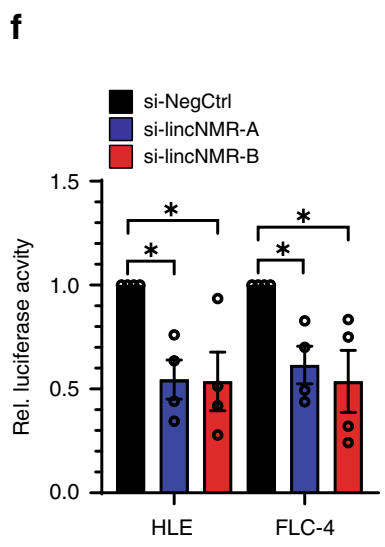

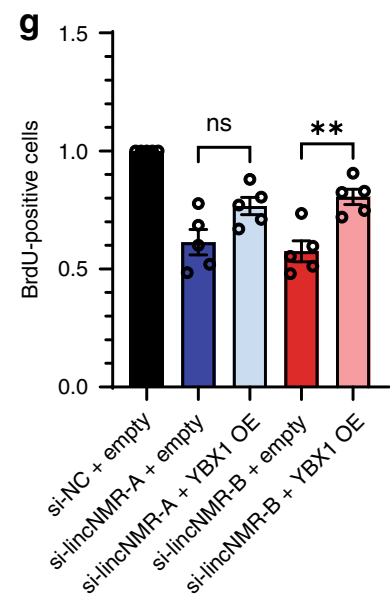

Fig. 3 lincNMR directly binds to and controls YBX1. a Volcano plot depicting total candidates $(n=701)$ identified by lincNMR in vivo RNA antisense purification followed by mass spectrometry (RAP-MS). In total, 48 candidates highlighted in red were selected for fold enrichment (FC $>2$ ) and adjusted $P$ value of enrichment $(P<0.001)$. XL: cross link. Significance was calculated by moderated $t$ test. $\mathbf{b}$ Interaction candidates identified by in vivo RAP-MS selected for fold enrichment in cross linked over non-cross linked samples ( $F C>2)$, adjusted $P$ - value of enrichment $(P<0.001)$, and sorted for log-rank $P$ - value in liver cancer patient survival (based on TCGA data). c While the overexpression of wild-type (WT) lincNMR partially rescues the decreased cell proliferation caused by lincNMR knockdown, a lincNMR mutant with a mutation in the first (A) of three (A, B, C) putative YBX1-binding sites is not capable of rescuing cell proliferation in HLE cells at $72 \mathrm{~h}$ post lincNMR depletion (assayed by BrdU incorporation, $n=4$ ). d Determination of RNA pull down efficiency in UV-RIP by RT-qPCR validating the interaction between YBX1 and lincNMR in HLE cells $(n=4)$. Data shown are normalized to SRSF4 as a negative control gene and control vector. e Silencing YBX1 with $10 \mathrm{nM}$ siPOOL imparts a proliferative disadvantage to HLE cells determined $72 \mathrm{~h}$ post transfection $(n=4)$. Data shown are normalized to negative control siPOOL. $\mathbf{f}$ Dual-luciferase assay for the transactivational activity of YBX1, which unravels the inhibition of YBX1 by lincNMR silencing. Data shown are the normalized ratios of YBX1-dependent Firefly luciferase activity divided by Renilla luciferase used for standardization after depletion of lincNMR with $10 \mathrm{nM}$ siPOOLs in HLE and FLC-4 cells $(n=4)$. $\mathbf{g}$ Overexpression of YBX1 partially rescues the proliferation defect induced by lincNMR depletion in HLE cells assayed by BrdU cell proliferation assay ( $72 \mathrm{~h}$ depletion of lincNMR and $66 \mathrm{~h}$ overexpression of YBX1). Data shown are normalized to empty vector transfected with negative control siPOOL $(n=5)$. $\mathbf{c}-\mathbf{g}$ Data represents mean and error bars represent SEM. Significance was calculated by unpaired, two-tailed $t$ test with ${ }^{\star} P<0.05 ;{ }^{\star \star} P<0.01$; ${ }^{\star \star \star} P<0.001$.

weight was also significantly reduced in lincNMR-depleted tumors in comparison with the control group (Fig. $2 \mathrm{~d}$ ).

lincNMR binds to YBX1 protein, which regulates cell survival. In order to gain insight into the molecular function and protein interaction partners of lincNMR, we performed in vivo RNA antisense purification (RAP-MS) ${ }^{41,42}$. After cross linking RNA and protein in vivo, we used biotinylated DNA oligos complementary to the lincNMR sequence to pull down lincNMR and its associated protein-binding partners from HLE cell lysates (Supplementary Fig. 4a). Pull down efficiency was confirmed by RT-qPCR with three different lincNMR amplicons, whereas PPIA and GAPDH mRNAs were used as negative controls (Supplementary Fig. 4b). Mass spectrometry analysis of proteins cross linked to and pulled down with lincNMR compared with non-cross linked lysates identified 701 proteins in total (Fig. 3a), with 48 proteins or isoforms enriched at least twofold and with an adjusted $P$-value $<0.001$ (Supplementary Data 2). To further select relevant interaction partners linked to
HCC, we analyzed their correlation to survival in HCC (Fig. 3b). In total, 22 candidates were significantly associated with the overall survival of liver cancer patients (Supplementary Data 2). YBX1 emerged as a potential binding partner with strongest and significant correlation to survival in liver cancer patients (Fig. 3b; Supplementary Fig. 4c). In addition, we searched for predicted RBP binding sites in the target genes using RBPmap ${ }^{43}$ and found three YBX1 sites and two SRSF3 sites $(P<0.001)$ in the lincNMR transcript. Mutation of the first of the three predicted consensus YBX1 sites in lincNMR (Supplementary Fig. 4d) abrogated the proliferation rescue of wild-type lincNMR (Fig. 3c). UV-RIP followed by RT-qPCR validated the interaction between lincNMR RNA and YBX1 protein. After immunoprecipitation of Flag-HA-tagged YBX1 (Supplementary Fig. 4e), lincNMR was enriched in the YBX1 pulldown compared with the negative control SRSF4 (Fig. 3d). In addition, biotinylated lincNMR RNA pulled down endogenous YBX1 protein in an in vitro RNA-affinity purification (Supplementary Fig. 4f). 
In addition to the significant association of high YBX1 expression with survival in hepatocellular carcinoma patients (Supplementary Fig. 4c), knockdown of YBX1 impaired cell proliferation by $50 \%$ (Fig. 3e) resembling the effect of lincNMR knockdown. LincNMR levels were decreased by $49 \%$ after YBX1 depletion (Supplementary Fig. 4g). Vice versa, YBX1 protein levels were decreased by $\sim 20 \%$ when lincNMR was silenced by two independent siPOOLs (Supplementary Fig. 4h). We also found a significant positive correlation between lincNMR and YBX1 mRNA in HCC patient samples (Supplementary Fig. 4i).

The direct interaction of lincNMR and YBX1 protein prompted us to assess the activity of YBX1 upon lincNMR depletion using luciferase assays for YBX1 transactivational activity. Knockdown of lincNMR significantly decreased the YBX1 activity in two independent liver cancer cell lines (Fig. 3f). In turn, overexpression of YBX1 partially rescued the proliferation deficit caused by lincNMR knockdown (Fig. 3g). Together, these data show that lincNMR interacts with and regulates YBX1, YBX1 mimics the impact of lincNMR on cell proliferation, and the regulation of lincNMR by YBX1 generates a feedforward loop leading to the correlation of expression in liver cancer.

lincNMR and YBX1 share target genes in nucleotide metabolism. To investigate the impact of lincNMR on the cellular proteome, we employed a triple-label stable isotope labeling by amino acids in cell culture (SILAC) approach (schematic Supplementary Fig. 5a). SILAC ratios were used for analysis comparing siPOOLs targeting lincNMR to negative control siPOOL (si-lincNMR-A/si-Neg Ctrl $=\mathrm{M} / \mathrm{L}$ and si-lincNMR-B/si-Neg $\mathrm{Ctrl}=\mathrm{H} / \mathrm{L}$ ). A correlation analysis served as a quality control on the complete dataset: a significant correlation was observed across three biological replicates and in between both siPOOLs targeting lincNMR with an average correlation coefficient of $R=0.74$ (Supplementary Fig. 5b). Notably, the correlation between replicates of the same siPOOL was slightly higher (range $0.69-0.87$ ) than between two different siPOOLs (range 0.43-0.54), which may point towards sequence-mediated off-target effects, but still indicates the high specificity and correlation $\left(P<10^{-100}\right)$ of the complex siPOOLs of 30 siRNAs compared with an average correlation of only 0.07 between individual siRNAs or lower complexity pools ${ }^{44}$.

For 242 candidates $(P$-value $<0.001)$ deregulated by both lincNMR-targeting siPOOLs in the same direction, a Panther Overrepresentation Analysis with FDR correction was performed using Reactome and PANTHER pathway datasets. This revealed a significant enrichment of key terms like "G1 / S transition" and "Cell Cycle Checkpoints" as well as "De novo pyrimidine deoxyribonucleotide biosynthesis" matching the phenotype observed after lincNMR depletion (Supplementary Fig. 5c). Among the strongly downregulated proteins upon lincNMR knockdown, we identified RRM2 (si-lincNMR-A $=-68 \%$, silincNMR-B $=-67 \%$ ), TK1 (si-lincNMR-A $=-52 \%$, si-lincNMR$\mathrm{B}=-58 \%$ ), and TYMS (si-lincNMR-A $=-43 \%$, si-lincNMR-B $=-57 \%$ ) and other key enzymes in nucleotide metabolism pathways (Fig. 4a, b), which were also part of the enriched gene ontology terms (Supplementary Fig. 5c). This decrease of RRM2, TK1, and TYMS proteins after lincNMR depletion was validated by western blotting (Fig. 4c) in good accordance with the independent triple-label SILAC-MS approach (Fig. 4d). In addition, a decrease of RRM2, TK1, and TYMS mRNAs was also observed after lincNMR depletion with both siPOOLs (Supplementary Fig. 5d). Since we identified lincNMR as a regulator of the transcription factor YBX1, we tested whether also YBX1 depletion would affect RRM2, TK1, and TYMS expression levels. Indeed, loss of YBX1 induced a significant decrease of RRM2, TK1, and
TYMS protein levels (Fig. 4e, f) establishing the lincNMRYBX1-RRM2 / TK1 / TYMS axis. Depletion of RRM2, TK1, and TYMS with siPOOLs significantly impaired cell proliferation mimicking the phenotype observed after lincNMR depletion (Fig. 5a) with a knockdown efficiency in the range of 95-99\% (Supplementary Fig. 5e). Similarly, the RRM2 inhibitor triapine ${ }^{45}$ induced an arrest of cell cycle progression in the G1 phase similar to lincNMR knockdown (Fig. 5b).

Knockdown of YBX1, TK1, or TYMS also significantly induced cellular senescence (Fig. $5 c$, d), further phenocopying the lincNMR knockdown. Knockdown of lincNMR, YBX1, TK1, and TYMS also significantly inhibited the colony-formation capacity of liver cancer cells (Fig. 5e, f). After the knockdown of RRM2, no cells were left precluding quantification of senescence or colony formation.

Next, we found RRM2, TK1, and TYMS to be strongly and significantly induced by about ten-, six- and fourfold, respectively, in liver cancer patient datasets from TCGA (Supplementary Fig. $6 \mathrm{a}-\mathrm{c}$ ). Furthermore, all three target genes, RRM2, TK1, and TYMS were significantly associated with poor survival in HCC patients (Supplementary Fig. $7 \mathrm{a}-\mathrm{c}$ ). The lincNMR expression level significantly positively correlated with RRM2, TK1, and TYMS mRNA levels in HCC patient samples (Supplementary Fig. 7d-f), further corroborating the strong link between lincNMR, these three regulators of nucleotide metabolism, and liver cancer.

Also, the expression of YBX1 mRNA significantly positively correlated with RRM2, TK1, and TYMS mRNA expression in 374 human hepatocellular carcinoma samples (TCGA, Fig. 6a-c). The YBX1 protein interacted with the promoter regions of the RRM2, TK1, and TYMS genes as revealed by chromatin immunoprecipitation (ChIP, Fig. 6d). Lastly, we performed luciferase assays with the promoter regions of RRM2, TK1, and TYMS fused to firefly luciferase. Knockdown of lincNMR or YBX1 significantly decreased the luciferase activity for all three promoters (Fig. 6e), further corroborating the regulatory interaction of lincNMR and YBX1 with RRM2, TK1, and TYMS.

Depletion of lincNMR leads to reduced dNTP levels. Since dNTP-synthesizing enzymes were downregulated by the knockdown of lincNMR, we further investigated whether the levels of dNTPs were accordingly affected. All four dNTPs, dATP, dCTP, dGTP, and dTTP, were significantly downregulated after lincNMR depletion with two independent siPOOLs in two independent cell lines (Fig. 7a, b). The knockdown of YBX1 recapitulated this phenotype also leading to decreased dNTP levels in two cell lines (Fig. 7c, d).

dNTPs rescue the lincNMR proliferation phenotype. Importantly, supplying exogenous dNTP pools by bathing the cells rescued the impact of lincNMR knockdown on cell proliferation by preventing this phenotype (Fig. 7e, f). This effect was dosedependent and statistically significant, illustrating the essential role of nucleotide metabolism in the pro-proliferative function of lincNMR.

In summary, their regulation, their association with survival, and their correlation of expression links lincNMR, YBX1, RRM2, TK1, and TYMS to liver cancer and to each other, respectively. LincNMR affects cell viability, proliferation, senescence, colony formation, and tumor growth in vivo. The interactor YBX1 and its targets RRM2, TK1, and TYMS mimic the phenotypes of lincNMR. At the molecular level, these data suggest a model (Fig. 7g) in which the lncRNA lincNMR binds to YBX1, increases its activity resulting in the upregulation of the enzymes RRM2, TK1, and TYMS, which mediate an increase in nucleotide 

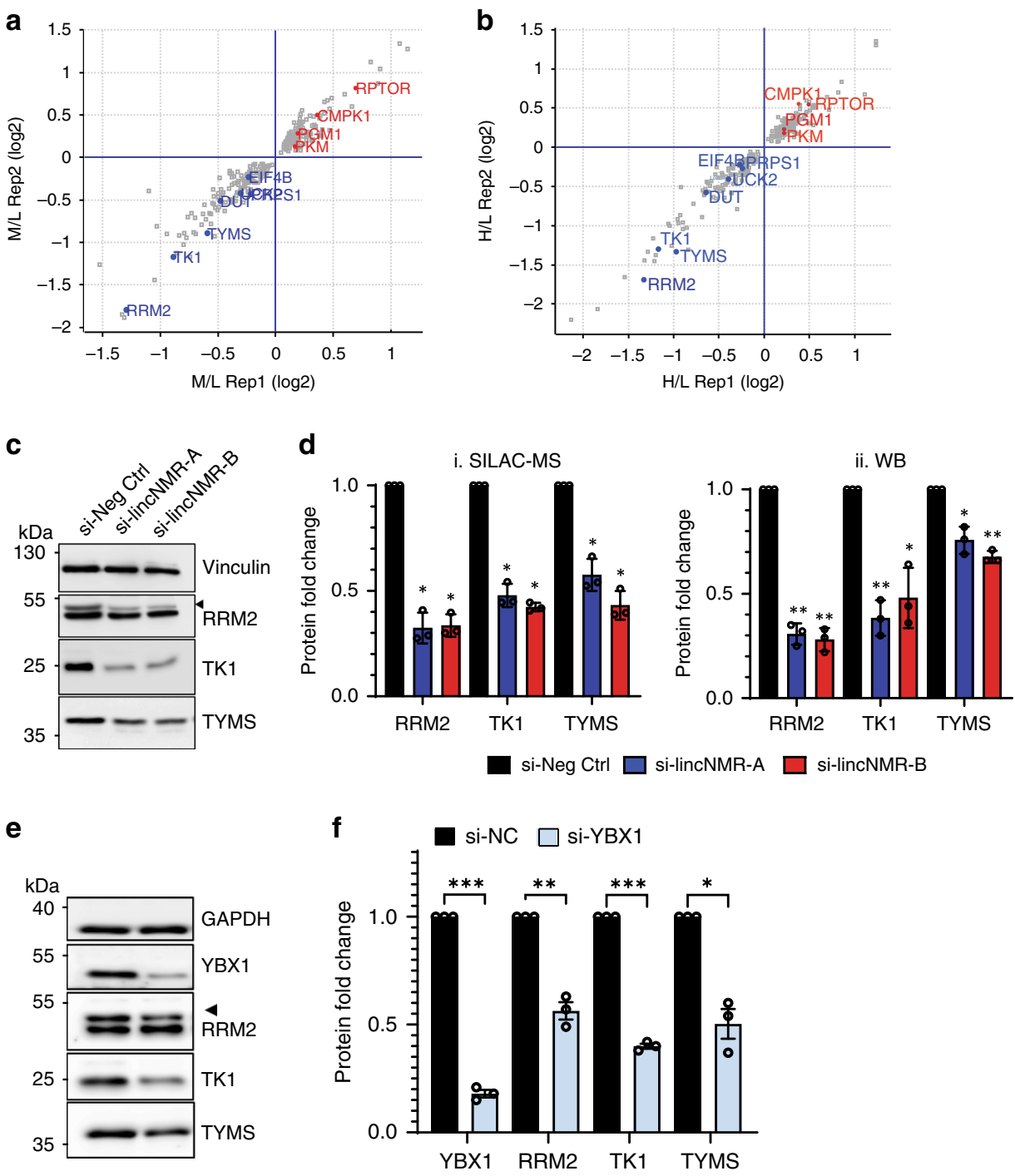

Fig. 4 lincNMR depletion downregulates key dNTP metabolism enzymes. a Triple-label SILAC-MS: scatter plot showing normalized M/L ratios representing deregulated proteins $48 \mathrm{~h}$ after lincNMR knockdown in replicates 1 and 2 with $10 \mathrm{nM}$ siPOOLs in HLE cells (M/L = si-lincNMR-A/si-Neg Ctrl). b Triple-label SILAC-MS: scatter plot showing normalized H/L ratios representing deregulated proteins $48 \mathrm{~h}$ after lincNMR knockdown in replicates 1 and 2 with $10 \mathrm{nM}$ siPOOLs in HLE cells (H/L = si-lincNMR-B/si-Neg Ctrl). c Western blot validation of SILAC-MS data depicting downregulation of RRM2, TK1, and TYMS proteins in HLE cells $72 \mathrm{~h}$ after lincNMR knockdown with $10 \mathrm{nM}$ siPOOLs $(n=3)$. Vinculin was used as a loading control. d Quantitative comparison of SILAC-MS (i) and western blot (ii) results confirming consistent downregulation of RRM2, TK1 and TYMS ( $n=3$ ). log2 fold change was calculated and normalized to negative control siPOOL. e YBX1 silencing inhibits the expression of RRM2, TK1, and TYMS at $72 \mathrm{~h}$ post transfection with 10 $\mathrm{nM}$ siPOOL in HLE cells documented by western blotting $(n=3)$. GAPDH was used as a loading control. $\mathbf{f}$ Quantification of western blot from (e): protein fold change was calculated by normalizing the RRM2, TK1, and TYMS signal to loading control GAPDH and to negative control siPOOL ( $n=3$ ). $\mathbf{a}$, $\mathbf{b}$ Data represent log2 fold change normalized to negative control siPOOL from two independent replicates. Highlighted proteins represent key deregulated players in purine and pyrimidine metabolism according to KEGG pathway annotations. Color key: red = upregulated proteins; blue = downregulated proteins. d, $\mathbf{f}$ Data represent mean, and error bars represent SEM. Significance was calculated by unpaired, two-tailed t test with ${ }^{\star} P<0.05$; ${ }^{\star \star} P<0.01 ;{ }^{\star \star \star} P<0.001$.

metabolism. In turn, lincNMR depletion causes a decrease of dNTPs leading to cellular senescence.

\section{Discussion}

In this study, we identified a lncRNA - lincNMR, a first lncRNA to regulate nucleotide metabolism in cancer cells. Silencing of lincNMR leads to impaired cell proliferation and colony formation, induction of a G0 / G1 phase cell cycle arrest, deregulation of nucleotide metabolism, and eventual induction of senescence in multiple cancer cell lines. LincNMR overexpression rescues the proliferation defect pointing toward the specificity of the knockdown.
Cellular senescence is defined by an irreversible cell growth arrest. While cells undergoing senescence do not replicate, they remain metabolically active and undergo alterations in cell metabolism pathways, including nucleotide, glucose, mitochondrial, and lipid metabolism ${ }^{46}$.

Nucleotide pools are essential for a multitude of biological processes, and their synthesis is carefully regulated during cell proliferation. dNTP pools are synthesized de novo or via the salvage pathway ${ }^{47}$. The rate-limiting step in the synthesis of dNTP pools is the reduction of ribonucleoside di- or tri-phosphates (NDPs / NTPs) to deoxyribonucleotide di- or tri-phosphates (dNDPs / dNTPs) by ribonucleotide reductase (RNR) ${ }^{48,49}$. 
a

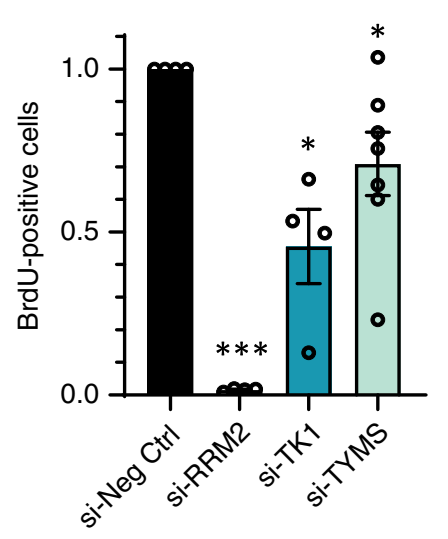

C

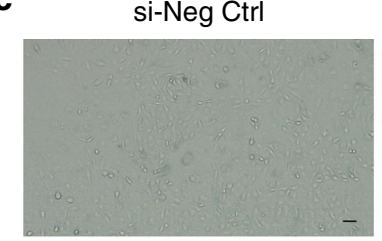

si-TK1

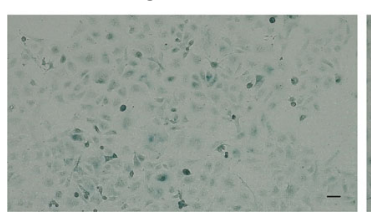

e

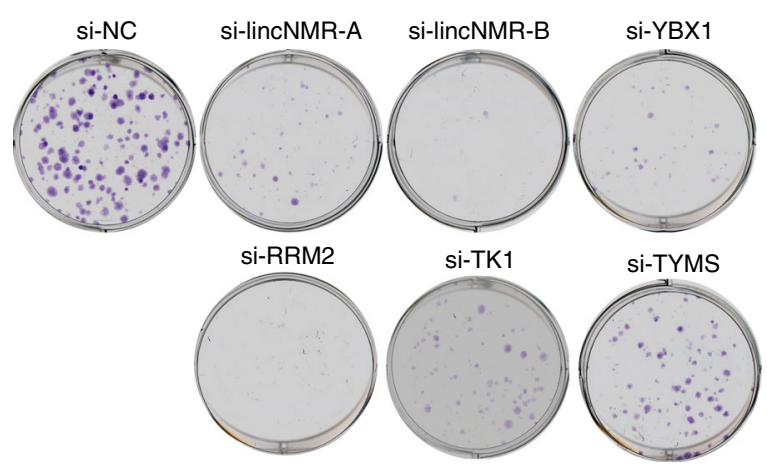

b

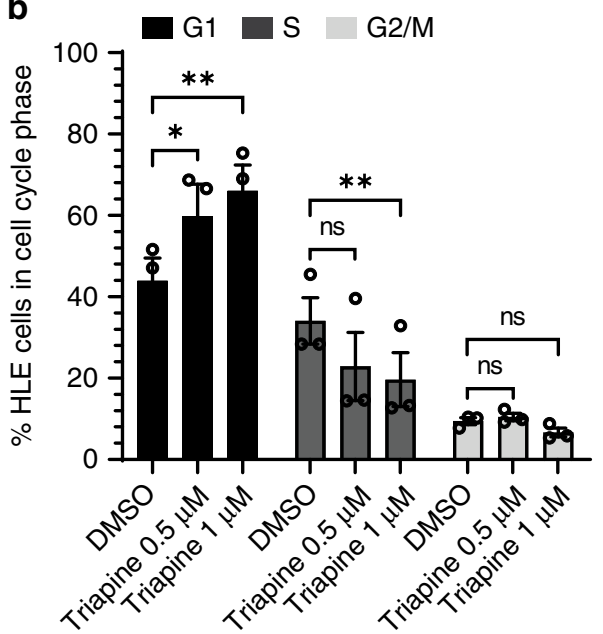

si-YBX1

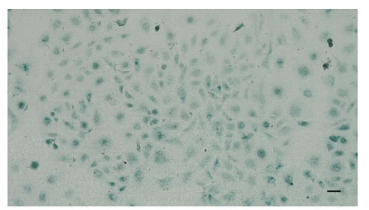

Si-TYMS

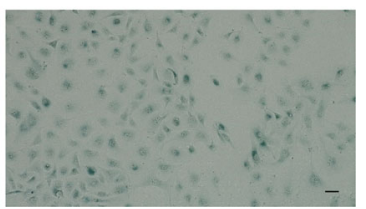

d

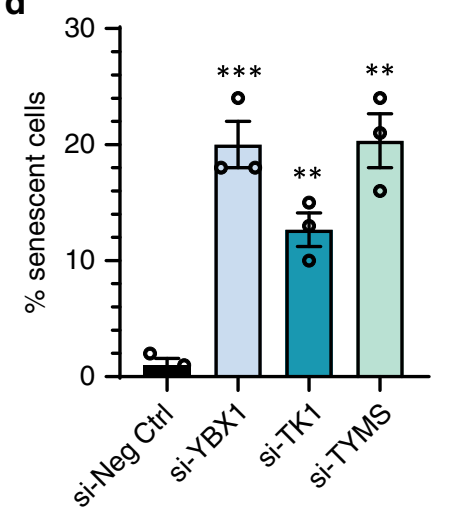

f

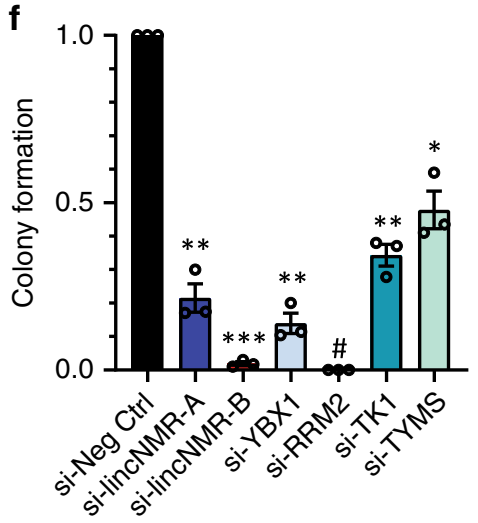

Fig. 5 Silencing YBX1, RRM2, TK1, or TYMS mimics the phenotype of lincNMR depletion. a Depletion of RRM2, TK1, and TYMS invokes a strong proliferation defect $72 \mathrm{~h}$ after knockdown using $10 \mathrm{nM}$ siPOOLs targeting RRM2, TK1, or TYMS in HLE cells in at least three biological replicates. b The RRM2 inhibitor Triapine $(0.5 \mu \mathrm{M}$ and $1 \mu \mathrm{M})$ induces cell cycle arrest in the GO / G1 phase at $72 \mathrm{~h}$ post treatment in HLE cells. DMSO was used as a control. Data analysis was performed using the cell cycle analysis platform in the FlowJo software v10 $(n=3)$. c Representative microscopic images showing increased $\beta$-Gal activity indicating induction of senescence in HLE cells at $96 \mathrm{~h}$ post knockdown with $10 \mathrm{nM}$ of the respective siPOOLs $(n=3)$. The scale bar represents $100 \mu \mathrm{m}$. d SA- $\beta$-Gal assay quantification: bar graph representing percent $\beta$-Gal-positive cells after depletion of YBX1, TK1, and TYMS in HLE cells $(n=3)$. RRM2-depleted cells were abolished, and hence not available for analysis. e Representative images showing the impact of silencing of lincNMR, YBX1, RRM2, TK1, and TYMS on colony-forming efficiencies at 10 days after transfection using $10 \mathrm{nM}$ siPOOLs in HLE cells $(n=3)$. f Quantification of colony formation after knockdown represented relative to the negative control siPOOL $(n=3)$. \#: Following RRM2 knockdown, no colonies were growing $(0)$ and hence, no statistical significance could be calculated. $\mathbf{a}, \mathbf{b}, \mathbf{d}, \mathbf{f}$ Data represent mean, and error bars represent SEM. Significance was calculated by unpaired, two-tailed $t$ test with ${ }^{\star} P<0.05 ;{ }^{\star \star} P<0.01 ;{ }^{\star \star \star} P<0.001$.

Deregulation of nucleotide metabolism has been reported to play a pathogenic role in various diseases, including cancer $^{50,51}$.

In our triple-label SILAC-MS, knockdown of lincNMR leads to strong downregulation of key enzymes essential for dNTP biosynthesis - RRM2 (ribonucleotide reductase subunit 2), TK1 (thymidine kinase), and TYMS (thymidylate synthetase) among other cell cycle regulators and proliferation markers. These are also induced in HCC, associated with poor survival, and correlated with lincNMR expression, and their knockdown phenocopies the effect of lincNMR on cell proliferation, senescence, and colony formation. 
a

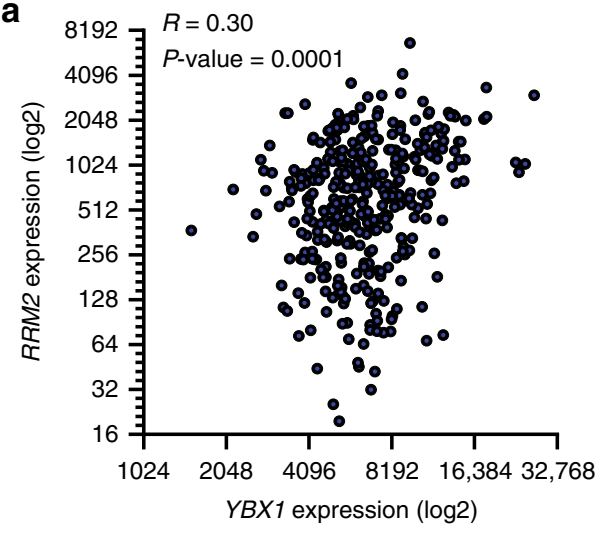

C

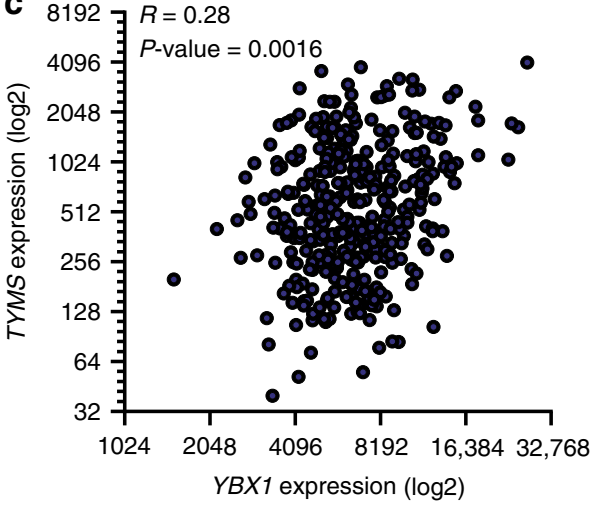

e

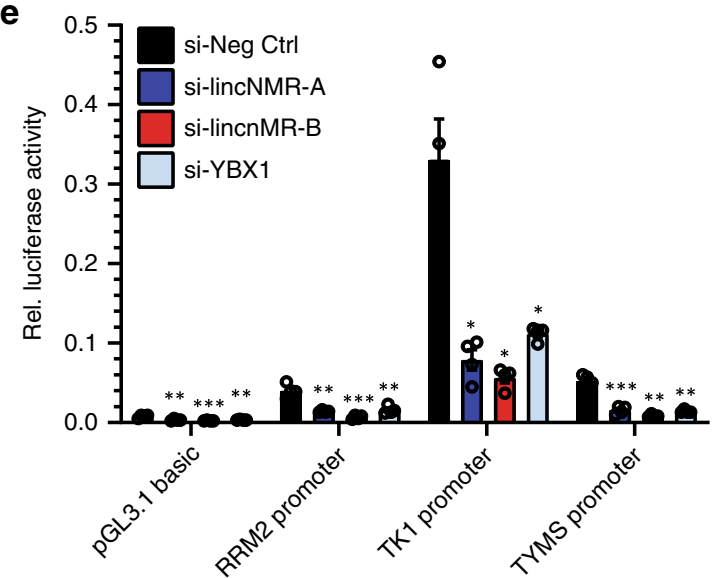

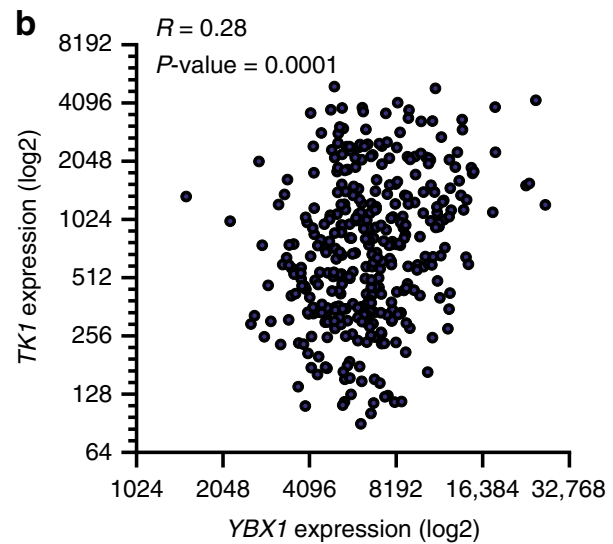

d

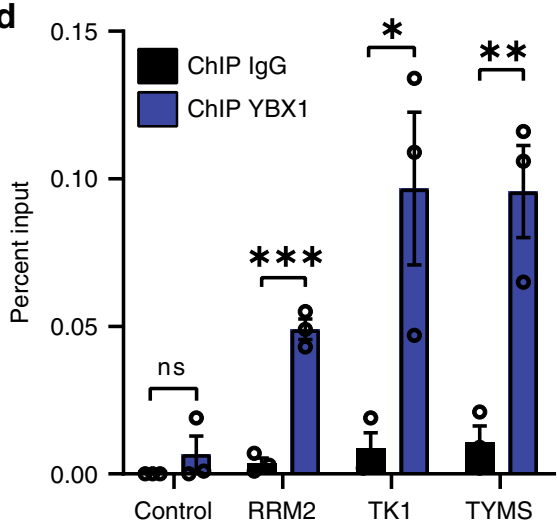

Promoter region coupled to luciferase

Fig. 6 YBX1 correlates with, binds to and activates the promoters of RRM2, TK1, and TYMS. a YBX1 mRNA expression significantly and positively correlates with RRM2 mRNA expression in $n=374$ hepatocellular carcinoma patient samples (TCGA LIHC, indicated: log2 expression). Significance was calculated by unpaired, two-tailed $t$ test. b YBX1 mRNA expression significantly and positively correlates with TK1 mRNA expression in $n=374$ hepatocellular carcinoma patient samples (TCGA LIHC, indicated: log2 expression). Significance was calculated by unpaired, two-tailed $t$ test. c YBX1 mRNA expression significantly and positively correlates with TYMS mRNA expression in $n=374$ hepatocellular carcinoma patient samples (TCGA LIHC, indicated: log2 expression). Significance was calculated by unpaired, two-tailed $t$ test. $\mathbf{d}$ The binding of YBX1 to the promoters of RRM2, TK1, and TYMS was determined by chromatin immunoprecipitation (ChIP) in HLE cells. Bar graph represents QPCR data after YBX1 ChIP compared with IgG as a negative control $(n=3)$. Data represent mean, and error bars represent SEM. Significance was calculated by unpaired, two-tailed $t$ test where ${ }^{\star} P<0.05$; ${ }^{\star \star} P<0.01$; ${ }^{* \star *} P<0.001$. e Luciferase assays show decreased transactivation of RRM2, TK1, and TYMS promoter regions after lincNMR or YBX1 silencing with $10 \mathrm{nM}$ siPOOLs in HLE cells $(n=4)$. Data shown are ratios of firefly luciferase divided by Renilla luciferase used for standardization. Data represent mean, and error bars represent SEM. Significance was calculated by unpaired, two-tailed $t$ test where ${ }^{\star} P<0.05 ;{ }^{\star \star} P<0.01 ;{ }^{\star \star \star} P<0.001$.

Consequently, all four dNTPs are depleted upon lincNMR knockdown, consolidating its role in the nucleotide metabolism axis. Our findings are in line with previous studies, finding levels of all four dNTPs significantly decreased during
OIS ${ }^{50-52}$ due to a suppression of RRM2 as a driver and not as an effect of cell cycle exit ${ }^{51}$. Depleting p53 or pRB, two key factors in senescence, does not affect lincNMR-controlled senescence in good accordance with a previous study showing 
a

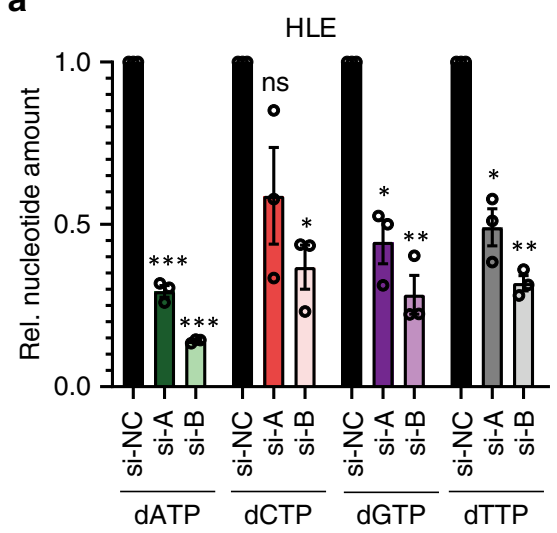

C

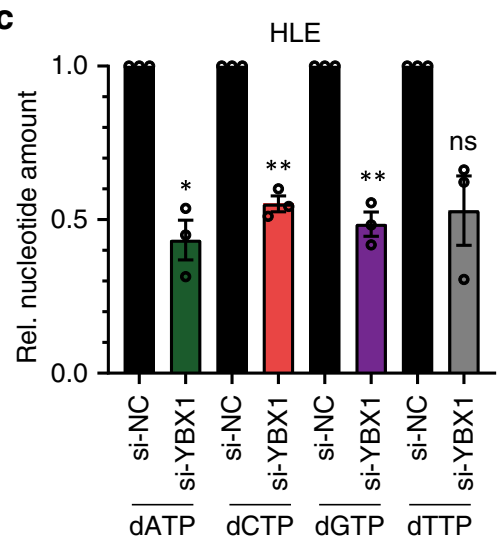

e

Schematic: dNTP bathing \& rescue assay

Day 1: transfect HLE with $10 \mathrm{nM}$ siPOOLs $\downarrow$

Day 2: bath cells in extracellular dNTPs

Day 3: cell proliferaon assay

g

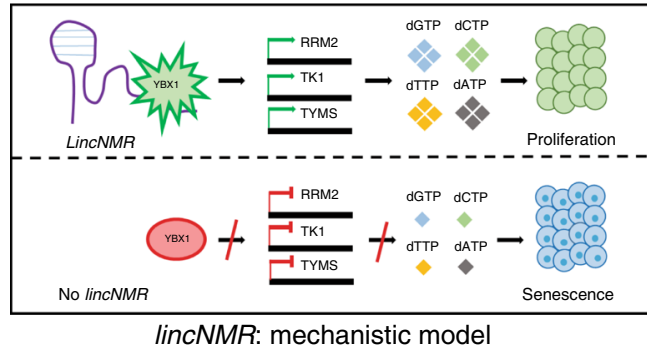

b

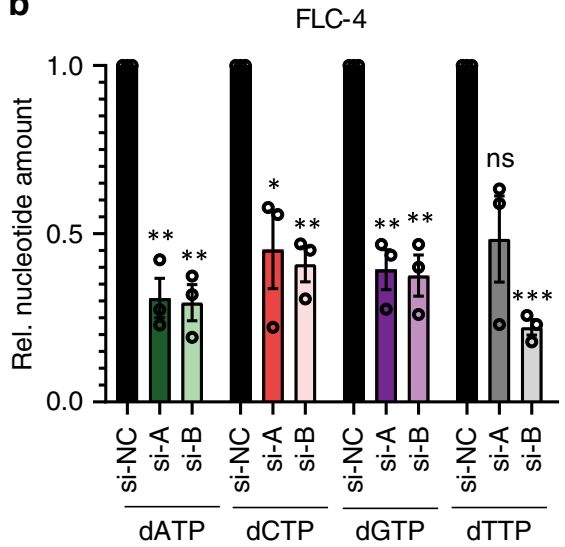

d

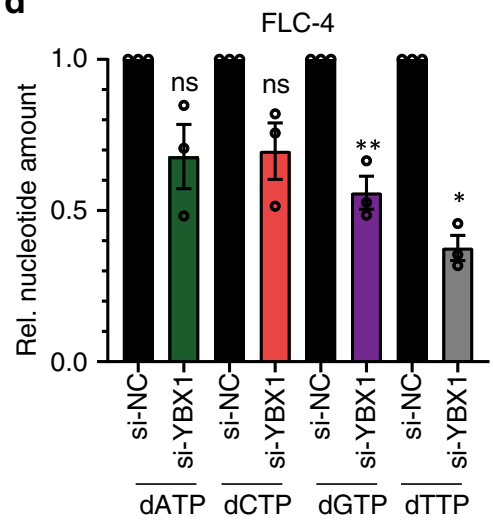

$\mathbf{f}$

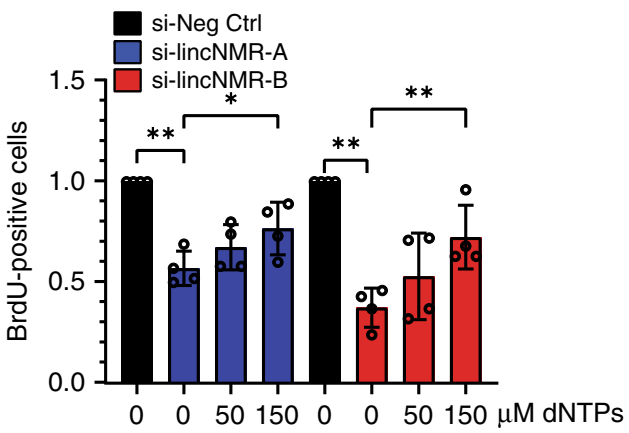

Fig. 7 LincNMR depletion reduces dNTP levels while supplying dNTPs rescues the lincNMR phenotype. a Quantification of dNTP levels uncovers that the depletion of lincNMR with $10 \mathrm{nM}$ siPOOLs leads to strong downregulation of dATP, dCTP, dGTP, and dTTP in HLE cells $(n=3)$. b Quantification of dNTP levels uncovers that the depletion of lincNMR with $10 \mathrm{nM}$ siPOOLs leads to strong downregulation of dATP, dCTP, dGTP, and dTTP in FLC-4 cells ( $n=3$ ). c Quantification of dNTP levels uncovers that the depletion of YBX1 with $10 \mathrm{nM}$ siPOOLs leads to downregulation of dATP, dCTP, dGTP, and dTTP in FLC-4 cells $(n=3)$. d Quantification of dNTP levels uncovers that the depletion of YBX1 with $10 \mathrm{nM}$ siPOOLs leads to downregulation of dATP, dCTP, dGTP, and dTTP in FLC-4 cells $(n=3)$. e Schematic outline of dNTP bathing \& rescue assay. $\mathbf{f}$ Supplying dNTPs rescues dose-dependently the proliferation decrease caused by lincNMR silencing determined by a BrdU incorporation assay. HLE cells were reverse transfected with $10 \mathrm{nM}$ of the respective siPOOLs and bathed in increasing concentrations $(0-150 \mu \mathrm{M})$ of extracellular pools of dNTPs $24 \mathrm{~h}$ post lincNMR depletion $(n=4)$. Data shown represent the results of the cell proliferation assay normalized to negative control siPOOL with the respective dNTP concentration. $\mathbf{g}$ Proposed model of lincNMR molecular mechanism. a-d, $\mathbf{f}$ Data shown are normalized to negative control siPOOL. Data represent mean, and error bars represent SEM. Significance was calculated by unpaired, two-tailed $t$ test with ${ }^{\star} P<0.05 ;{ }^{\star \star} P<0.01 ;{ }^{\star \star \star} P<0.001$. 
that senescence induced by RRM2 depletion is independent of p53 and $\mathrm{pRB}^{51}$.

Importantly, bathing cells in exogenous dNTP pools dosedependently rescues the proliferation phenotype caused by lincNMR knockdown in two liver cancer cell lines, which is in good accordance with previous studies stating that increasing dNTP levels by RRM2 overexpression or exogenous nucleoside supply overcomes aberrant DNA replication, DNA damage, and senescence induced by oncogenic RAS or BRAF ${ }^{51,52}$. Ectopic coexpression of TYMS and RRM2 also suppresses OIS in normal human fibroblasts ${ }^{52,53}$. TYMS and RRM2 are suppressed in cMYC-depleted melanoma cells undergoing OIS, and this senescent effect is rescued by overexpression of TYMS and RRM2 or by addition of deoxyribonucleosides ${ }^{54}$.

To gain an insight into the molecular mechanism, we identified YBX1 as a direct binding partner of lincNMR in vivo and in vitro. YBX1 is a transcription factor and also binds to lncRNAs linked to cancer ${ }^{55-58}$. YBX1 drives tumorigenicity and invasiveness of melanoma cells and its expression represents a negative prognostic factor in primary melanoma patients ${ }^{59}$. YBX1 expression correlates with poor outcomes in breast cancer patients ${ }^{60}$. Accordingly, we find high YBX1 expression correlated with poor overall survival in liver cancer, as well. LincNMR and YBX1 mRNA expression significantly correlate in liver cancer patient samples as well as with RRM2, TK1, and TYMS mRNA expression. Importantly, YBX1 depletion mimics the lincNMR depletion phenotype in decreasing cell proliferation, inducing senescence, diminishing colony formation, affecting RRM2, TK1, and TYMS levels, decreasing dNTP levels and YBX1 also partially rescues the growth-inhibitory effect of lincNMR knockdown. YBX1 binds to the promoter regions of RRM2, TK1, and TYMS. LincNMR controls the transactivational activity of YBX1- hence, we report a role for the lincNMR-YBX1 axis in regulating nucleotide metabolism in liver cancer cells. Nonetheless, future studies will unravel whether lincNMR primarily acts via recruiting, regulating, or activating YBX1, and these data also do not exclude that lincNMR may have additional functions and relevant interactors.

While the findings that lincNMR RNA interacts with YBX1 protein, that it shares the same target genes RRM2, TK1, and TYMS, that lincNMR, YBX1, RRM2, TK1, and TYMS share the same loss-of-function phenotype regarding cell proliferation, senescence, and colony formation, that YBX1 expression or exogenous dNTPs rescue the proliferation defect caused by lincNMR depletion, and that lincNMR and YBX1 depletion reduces the transactivation of RRM2, TK1, and TYMS promoter fragments in luciferase assays, arguing in favor of a direct effect of the lincNMR-YBX1 axis, future research may also investigate secondary effects by cell cycle disturbance or by an impact on YBX1 regulation.

The copy number with a conservatively approximated minimum range of two to seven copies per cell on average classifies lincNMR as a moderately expressed lncRNA while other functional lncRNAs show much lower copy numbers ${ }^{61}$. On the one hand side, this may already be sufficient to recruit YBX1 to specific loci in the genome - on the other hand, this is likely an underestimation since the comparison is necessarily done to plasmid DNA, so the efficiency of RNA isolation and reverse transcription is assumed to be quantitatively complete. Furthermore, the copy number of lincNMR could vary between different cell states (e.g., cell cycle, heterogeneity) and hence reach higher numbers for activity.

Depletion of lincNMR not only reduces proliferation in liver cancer but also impairs proliferation in multiple breast and lung cancer cell lines. In addition, it is overexpressed in multiple cancer entities like liver, lung, breast, bladder, and cervical cancer making it a likely broadly relevant oncogenic lncRNA. Xenograft experiments using $\operatorname{lin} c N M R$-depleted cells in the CAM model reveal the impact of lincNMR on tumor size in vivo potentially implicating it as a therapeutic target in the future.

Notably, targeting nucleotide metabolism via ribonucleotide reductase inhibitors has been identified as a promising therapeutic strategy in multiple cancer types. Ribonucleotide reductase (RNR) inhibitors show promise in the clinic for treating multiple cancer types with some even serving as a first-line cancer treatment. Gemcitabine is the first nucleoside analog clinically approved, and it continues to be a frontline therapy against pancreatic, bladder, and lung cancer ${ }^{62}$. Clofarabine is the second approved drug targeting refractory pediatric leukemia ${ }^{63,64}$. A combination therapy with gemcitabine, clofarabine, and carboplatin significantly improves progression-free survival of patients with platinum-sensitive recurrent ovarian cancer ${ }^{65}$. Another RNR inhibitor, hydroxyurea (HU), is used in treatment of AML, CML, and glioblastomas ${ }^{66-68}$.

Overall, our study identified a tumor-promoting lincRNA - $\operatorname{lin} c N M R$ - and unveils its mechanism along a YBX1-RRM2 / TK1 / TYMS axis in regulating nucleotide metabolism and governing the cancer cell fate between proliferation and senescence.

\section{Methods}

Cell culture. Liver cancer cell lines (HLE, HLF, FLC-4, and SNU-387) used in this study were kindly provided by Dr. Kai Breuhahn (Institute of Pathology, University Heidelberg, Heidelberg Germany). Lung cancer cell lines (A549, NCI-H1299, and NCI-H460) and breast cancer cell lines (MCF-7, KPL-1, and T47D) were purchased from ATCC. Liver cancer cells were cultured in RPMI-1640 (SigmaAldrich, R8758) with 10\% FBS. Lung and breast cancer cell lines were cultured in DMEM (Sigma-Aldrich, D5671) supplemented with 10\% FBS. All cell lines were cultured in a cell culture incubator at $37^{\circ} \mathrm{C}$ and $5 \% \mathrm{CO}_{2}$ without addition of any antibiotics. Cell lines were periodically tested at a 3-month interval for mycoplasma contamination using a PCR-based detection kit (PromoCell, PK-CA91-1048). All cell lines used in this study were verified using cell authentication services from Multiplexion, Heidelberg, Germany ${ }^{69}$.

siPOOL and plasmid transfections. siPOOLs were obtained as complex pools of 30 siRNAs targeting the same gene from siTOOLs Biotech $\mathrm{GmbH}$, Martinsried, Germany, to minimize off-target effects ${ }^{33}$. siPOOLs were reverse transfected using Lipofectamine RNAiMAX transfection reagent (Life Technologies, 133778150) with RPMI-1640 medium. Plasmids were transfected using a forward transfection protocol by using TurboFect transfection reagent (Fischer Scientific, R0531) and Opti-MEM (Gibco, 31985054). Transfections were performed according to the manufacturer's recommendations. Sequences of all siPOOLs used in this study are provided in Supplementary Data 3.

The double knockdown of TP53 and pRb was performed as described ${ }^{70}$. siPOOL sequences are listed in Supplementary Data 3.

RNA isolation, reverse transcription, and quantitative PCR. Cells were lysed in Trizol (Sigma, T9424-200ML), RNA isolation and DNase I (Roche, 4716728001) digestion were performed as per the manufacturer's instructions. RNA isolation for in vivo RAP-MS and UV - RIP qPCR experiments was performed as per the manufacturer's protocol using the miRNeasy Mini kit (Qiagen, 217004), and DNase digestion was performed using the TURBO DNAfree kit (Life Technologies, AM1907).

In total, $1 \mu \mathrm{g}$ of RNA was reverse transcribed using random hexamer primers with RevertAid reverse transcriptase (ThermoFisher, EP0442). cDNA was diluted 1:40 with DNase- and RNase-free water, and $4 \mu \mathrm{l}$ was used in a qPCR reaction. RT qPCR was performed using Power SYBR Green PCR Master Mix (ThermoFisher, 4367659) in an Applied Biosystems StepOne Plus thermal cycler with holding stage of $95^{\circ} \mathrm{C}$ for $10 \mathrm{~min}$ followed by 40 cycles of $95^{\circ} \mathrm{C}$ for $15 \mathrm{~s}$ and $60{ }^{\circ} \mathrm{C}$ for $30 \mathrm{~s}$. Normalization was performed with PPIA, GAPDH, and / or SRSF4 as internal reference controls as indicated. Data were analyzed using StepOne Software v2.3. Primer sequences used in this study are provided in Supplementary Data 4.

Protein isolation, protein quantification, and western blot. Cells were briefly washed with $1 \times$ PBS and lysed in $200 \mu \mathrm{l}$ RIPA buffer ( $50 \mathrm{mM}$ Tris-HCl pH 7.5, 150 $\mathrm{mM} \mathrm{NaCl}, 1 \%$ Triton X-100, 0.5\% Na-Deoxycholate, $0.1 \%$ SDS, $1 \%$ DTT) supplemented with $1 \times$ Protease (Roche, 4693132001) and phosphatase inhibitors (Sigma-Aldrich, 4906837001) on ice for $30 \mathrm{~min}$ with intermittent mixing. The lysate was centrifuged at $17,000 \mathrm{~g}$ for $10 \mathrm{~min}$ at $4{ }^{\circ} \mathrm{C}$ for clearing. The supernatant was transferred to new tubes and flash-frozen until further analysis. Protein quantification was performed using the Pierce BCA Protein Assay Kit (Thermo 
Fischer, 23224) as per the manufacturer's instruction. Samples were boiled for 10 min at $95^{\circ} \mathrm{C}$ in $4 \times$ Laemmli Buffer $(0.25 \mathrm{M}$ Tris $\mathrm{pH} 6.8,20 \%$ glycerol, $10 \%$ SDS, $355 \mathrm{mM}$ 2-mercaptoethanol, $0.002 \%$ bromophenol blue) before loading onto $10 \%$ SDS-PAGE gels. Proteins were transferred to nitrocellulose membranes using transfer buffer $(0.25 \mathrm{M}$ Tris-base, $1.92 \mathrm{M}$ Glycine, $1 \%$ SDS with $20 \%$ methanol) at $120 \mathrm{~V}$ for $90 \mathrm{~min}$. The membrane was blocked with $5 \%$ milk in $1 \times$ TBS-T $(247 \mathrm{mM}$ Tris, $1.37 \mathrm{M} \mathrm{NaCl}, 26.8 \mathrm{mM} \mathrm{KCl}, 0.05 \%$ Tween-20) for $30 \mathrm{~min}$. Blots were incubated with the primary antibodies in $5 \%$ milk in $1 \times$ TBT-T overnight at $4{ }^{\circ} \mathrm{C}$ with indicated dilutions. After incubation with primary antibody, blots were washed five times with $1 \times$ TBS-T for $5 \mathrm{~min}$ at room temperature on a shaker. Next, blots were incubated for $1 \mathrm{~h}$ at room temperature with respective anti-mouse or anti-rabbit HRP-conjugated secondary antibodies (Jackson ImmunoResearch Laboratories, 115-035-003 and 111-035-144) were used at a dilution of 1:2500. After incubation with secondary antibody, blots were washed again for five times with $1 \times$ TBS-T for $5 \mathrm{~min}$ at room temperature on a shaker. Membranes were developed using Supersignal Pico (Fisher Scientific, 34580). Images were acquired on the Intas ChemoCam Imager, and signal quantification was performed using LabImage 1D software. A list of antibodies and dilutions used is provided in Supplementary Data 5.

Cell viability. CellTiter-Glo Luminescent Cell Viability Assay (Promega kit, G7572) was performed $72 \mathrm{~h}$ after knockdown with the respective siPOOLs. At the time point for the assay, growth medium was removed from the cells using multichannel pipette and $60 \mu \mathrm{L}$ of 1:4 CellTiter-Glo reagent: $1 \times$ PBS was added to the cells. Plate was incubated for $15 \mathrm{~min}$ at room temperature in the dark using an orbital shaker. After the incubation, chemiluminescence was measure using luminometer (Fluoroskan Ascent FL, Thermo Scientific). Data obtained were normalized to siPOOL-negative control.

Rapid amplification of CDNA ends (RACE). The total RNA (treated with DNase I) from HLE cells was used for first-strand cDNA synthesis. The SMARTer RACE cDNA Amplification Kit (Clontech, 634923) was used to perform 5' - and 3'-RACE analysis according to the manufacturer's instructions. The gene-specific primers used for RACE are listed in Supplementary Data 6.

Subcellular fractionation. Subcellular fractionation was performed in HLE cells to separate chromatin-associated, nucleoplasmic and cytoplasmic fractions as previously described ${ }^{71}$. Fraction-specific controls were used to assess the quality of fractions obtained (chromatin fraction: NEAT1, MALAT1; nucleoplasmic fraction: $R N U-1$; cytoplasmic fraction: DANCR). Primer sequences are provided in Supplementary Data 4.

Cell proliferation. BrdU incorporation efficiency of cells was measured at $72 \mathrm{~h}$ post knockdown with respective siPOOLs using the Cell Proliferation Assay kit (Roche, $11669915001)$ as per the manufacturer's instructions.

Cell cycle analysis. Seventy-two hours post treatment with siPOOLs, the cells were trypsinized and fixed in $70 \%$ ethanol overnight at $-20^{\circ} \mathrm{C}$. Fixed cells were pelleted and washed with $1 \times$ PBS. After washing, cells were resuspended in $1 \times$ PBS containing $100 \mu \mathrm{g} / \mathrm{ml}$ RNase A (Sigma, 10109169001) and incubated at $37^{\circ} \mathrm{C}$ for $30 \mathrm{~min}$. Post RNase treatment, the cells were stained with $100 \mu \mathrm{g} / \mathrm{ml}$ propidium iodide (Sigma-Aldrich, P4170). In total, 10,000 cells were acquired on BD FACSCanto II Flow Cytometer, and data analysis was performed using FlowJo v10 software.

Triapine (Selleckchem, S7470), the RRM2 inhibitor ${ }^{45}$, was dissolved in DMSO, and HLE cells were treated with respective concentrations. Cell cycle analysis was performed as described, and data were analyzed using FlowJo software.

Apoptosis Caspase-Glo assay. The assay was performed using the Caspase-Glo assay kit from Promega (G8091) in a 96-well format. Cells were transfected with 10 $\mathrm{nM}$ of respective siPOOLs and incubated at $37^{\circ} \mathrm{C}$ with $5 \% \mathrm{CO}_{2}$ for $72 \mathrm{~h}$. The supernatant was discarded and replaced with diluted (1:2 in $1 \times$ PBS) Caspase-Glo solution. The plate was incubated for $60 \mathrm{~min}$ at RT in the dark. Luminescence was measured with a FLUOstar Omega microplate reader (BMG Labtech).

Senescence-associated $\boldsymbol{\beta}$-Gal assay. The Cells were reverse transfected with the respective siPOOLs on a six-well plate, and SA- $\beta$-Gal activity was detected $96 \mathrm{~h}$ post transfection. Cells were washed with $1 \times$ PBS and fixed at room temperature with $0.5 \%$ glutaraldehyde for $20 \mathrm{~min}$. Cells were washed twice with $1 \times$ PBS supplemented with $1 \mathrm{mM} \mathrm{MgCl} 2$ (pH 6.0) for 10 min on a rocker. In all, $2 \mathrm{ml} \mathrm{X-Gal}$ staining solution ( $1 \times \mathrm{PBS}$ containing $1 \mathrm{mM} \mathrm{MgCl} 2,41 \mathrm{mg}$ of potassium hexacyanoferrate (III), $52.5 \mathrm{mg}$ of potassium hexacyanoferrate (II) trihydrate, $1 \mathrm{mg} / \mathrm{ml}$ $\mathrm{X}$-Gal (5-bromo-4-chloro-3-indolyl-beta-D-galacto-pyranoside), $\mathrm{pH}$ 6.0) was added, and the dishes were sealed with parafilm and incubated overnight at $37^{\circ} \mathrm{C}$. Next day, the cells were washed three times with distilled water, and microscopy pictures were taken using a 10x objective of a Zeiss Cell Observer microscope. For analysis, 100 cells were counted, and percent senescent cells per condition are depicted in the bar graph.
Chick chorioallantoic membrane (CAM) assay. Pathogen-free fertilized eggs were purchased from Valo Biomedia $\mathrm{GmbH}$ and incubated in an incubation oven with $60 \%$ humidity at $37^{\circ} \mathrm{C}$. On day 8 , lincNMR was knocked down with $10 \mathrm{nM}$ siPOOLs using a forward transfection protocol described before in HLE cells. On day 9, eggs were windowed with an electric drilling tool. On the same day, in parallel, $1 \times 10^{6}$ transfected HLE cells were resuspended in $10 \mu \mathrm{L}$ growth media, mixed with $10 \mu \mathrm{L}$ Matrigel (Corning, 354262) and incubated for $10 \mathrm{~min}$ at $37^{\circ} \mathrm{C}$ and $5 \% \mathrm{CO}_{2}$. The cell-matrigel mix was seeded onto the $\mathrm{CAM}$, and the window was sealed for five days and incubated at $37^{\circ} \mathrm{C}$ with $60 \%$ humidity. During these days, eggs were observed, and dead or injured embryos were excluded from further experiments. On day 6, after seeding the cells on the CAM, chicken embryos were euthanized by a quick decapitation, and tumors were harvested from the CAM. Harvested tumors were processed, cleaned, and collected in ice-cold 1× PBS Images of tumors from HLE cells transfected with negative control siPOOL, and two independent siPOOLs targeting lincNMR were taken. Tumors were weighed on a microscale.

Colony formation assay. Colony formation assays were performed as described in ref. ${ }^{72}$. In brief, HLE cells were reverse transfected with $10 \mathrm{nM}$ of respective siPOOLs. Twenty-four hours later, 500 cells were reseeded into six-well plates to allow formation of colonies for 14 days. After the incubation, cells were fixed with $6 \%$ glutaraldehyde and stained with $1 \%$ crystal violet solution. After subsequent washes, plates were allowed to dry at room temperature, and colonies were counted.

In vivo RNA antisense purification (in vivo RAP-MS). Biotinylated DNA oligos complementary to lincNMR sequence were ordered from IDT, sequences are available in Supplementary Data 7. One billion HLE cells were used per pulldown per condition per biological replicate. In vivo RAP-MS was performed as per the protocol described previously ${ }^{41}$.

Generation of plasmids and mutagenesis. Gateway entry vectors were obtained from the DKFZ plasmids and clone repository. LincNMR-001 was amplified using the primers listed in Supplementary Data 10. Gateway LR reaction was performed with 50-150 ng of entry vector using LR Clonase II (Thermo, 11791020) as per the manufacturer's instructions into the gateway destination vector $\mathrm{pFRT}$-Flag/HA. Machl cells were used for transformation. Mini-Prep was performed using the NucleoSpin ${ }^{\circledast}$ Plasmid kit (Macherey \& Nagel, 740588.250). Midi-Prep was done using the PureLink ${ }^{\mathrm{mm}}$ HiPure Plasmid kit (Invitrogen, K210004). Services from Eurofins genomics/GATC were used for sequencing with CMV.for CGCAAATGG GCGGTAGGCGTG and BGH.rev TAGAAGGCACAGTCGAGG primers. Finally, cells were transfected with respective plasmid and empty vector pFRT-Flag-HA$\Delta \mathrm{CmR}-\Delta \mathrm{ccdB}$ as a control plasmid. Overexpression was confirmed by western blot using anti-Flag-M2 or anti-HA antibody (Supplementary Data 5).

Mutagenesis of the predicted high-confidence YBX1-binding sites (RBPmap) in the lincNMR transcript was performed using Phusion DNA polymerase as per supplier's instructions (NEB, M0530S). Primers used for performing PCR are listed in Supplementary Data 10.

UV cross linking RNA immunoprecipitation (UV-RIP) assay. Cell Seeding, Transfection, UV Cross linking, harvesting: on day $1,4 \times 10^{6} \mathrm{HLE}$ cells were seeded onto a $15-\mathrm{cm}$ dish. On day 2, $2 \mu \mathrm{g}$ of the respective constructs (Empty vector plasmid pFRT-Flag-HA- $\Delta$ CmR- $\Delta c c d B$ or pFRT-Flag/HA-YBX1) were transfected using TurboFect transfection reagent with a forward transfection protocol. On day 4, cells were UV cross linked at a wavelength of $254 \mathrm{~nm}$ using $0.8 \mathrm{~J} / \mathrm{cm}^{2}$ (instrument setting: $8000 \times 100 \mu \mathrm{J} / \mathrm{cm}^{2}$ ) and then lysed in high-strength cell lysis buffer (10 mM Tris- $\mathrm{HCl} \mathrm{pH}$ 7.5, $500 \mathrm{mM}$ lithium chloride, $0.5 \%$ dodecyl maltoside, $0.2 \%$ SDS, $0.1 \%$ sodium deoxycholate) supplemented with inhibitors cocktail containing SUPERase In (Thermo Scientific, AM2696), protease and phosphatase inhibitors. Lysate was passed through a syringe to break up the pellet, and DNase digestion was performed as per the manufacturer's instruction using the Turbo DNA-free Kit (Ambion, AM1907). Protein quantification was performed using the BCA reagent, and overexpression of YBX1 was confirmed by probing with anti-HA antibody on a western blot.

Pulldown: $1.5 \mu \mathrm{g}$ of cell lysate was used to perform UV- RIP using anti-Flag magnetic beads, and IP was confirmed by probing for anti-HA using western blot. For UV-RIP, $150 \mu \mathrm{l}$ of anti-Flag magnetic beads were used per pulldown. Beads were prewashed $5 \times$ using $1 \times$ TBS and resuspended in supplemented cell lysis buffer. In total, $5 \%$ lysate was removed for the Input fraction, and $150 \mu \mathrm{l}$ of prewashed beads were added to the cell lysate and incubated for $1 \mathrm{~h}$ at $4{ }^{\circ} \mathrm{C}$ with rotation. After the incubation, beads were magnetically separated. $50 \mu \mathrm{l}$ of flowthrough was saved to confirm the depletion of YBX1, and the remaining flowthrough was discarded. Beads were washed $2 \times$ with low salt-wash buffer $(0.1 \%$ SDS, $0.5 \%$ sodium deoxycholate, $0.5 \% \mathrm{NP}-40,0.01 \mathrm{M} \mathrm{NaCl}, 0.002 \mathrm{M} \mathrm{KCl}, 0.001 \mathrm{M}$ $\left.\mathrm{Na}_{2} \mathrm{HPO}_{4}, 0.0001 \mathrm{M} \mathrm{KH}_{2} \mathrm{PO}_{4}\right)$ and then with high salt-wash buffer $(0.1 \%$ SDS, $0.5 \%$ sodium deoxycholate, $0.5 \% \mathrm{NP}-40,0.05 \mathrm{M} \mathrm{NaCl}, 0.010 \mathrm{M} \mathrm{KCl}, 0.005 \mathrm{M}$ $\mathrm{Na}_{2} \mathrm{HPO}_{4}, 0.0005 \mathrm{M} \mathrm{KH}_{2} \mathrm{PO}_{4}$ ) with rotation for 5 min per wash. Beads were magnetically separated and resuspended in $500 \mu \mathrm{l}$ of RNase-free pure water. Beads were then separated into $20 \%$ (for protein extraction) and $80 \%$ (RNA isolation) to 
confirm the IP and lincNMR pulldown, respectively. Antibodies and magnetic beads used are listed in Supplementary Data 5.

Elution of RNA: RNA was eluted from the beads by reversal of UV cross linking in high salt buffer combined with Proteinase K digestion $(160 \mu$ l of Proteinase K buffer (125 mM Tris- $\mathrm{HCl} \mathrm{pH} \mathrm{7.8,62.5} \mathrm{mM} \mathrm{NaCl,} 12.5 \mathrm{mM}$ EDTA) and $40 \mu \mathrm{l}$ of Proteinase K) by incubating at $37^{\circ} \mathrm{C}$ with shaking for $30 \mathrm{~min}$ at $1000 \mathrm{rpm}$. Next, after addition of $500 \mu \mathrm{l}$ of Trizol to the tube, the tube was vortexed for $10 \mathrm{~s}$ and stored at $-20^{\circ} \mathrm{C}$ until ready for RNA isolation. RNA isolation, reverse transcription, and qPCR were performed as described above.

Elution of proteins: Captured, washed beads were boiled with $1 \times$ SDS loading buffer at $95^{\circ} \mathrm{C}$ for $10 \mathrm{~min}$, and western blot was performed with input, supernatant, and flow-through samples to confirm the YBX1 pulldown.

In vitro lincNMR RNA-affinity purification. In vitro lincNMR RNA-affinity purification was performed as described previously ${ }^{73}$. The lincNMR sequence (1100 nt) was cloned into a pcDNA3.1 vector, which was further linearized using EcoRV and MluI digestion. As a negative control, the lncRNA HULC (560 nt) was used for comparison, which had been previously cloned ${ }^{73}$. The MEGAscript T7 Transcription Kit (Ambion, AMB13345) was used according to the manufacturer's instructions for in vitro transcription. In vitro transcribed biotinylated lncRNAs were used to pull down the interacting proteins from HLE cell lysate using streptavidin sepharose beads (GE Healthcare, 17-5113-01). After subsequent washing steps, proteins were eluted using wash buffer (20 mM HEPES pH 7.9, 300 $\mathrm{mM} \mathrm{KCl}, 10 \mathrm{mM} \mathrm{MgCl} 2,0.01 \% \mathrm{NP}-40,1 \mathrm{mM}$ DTT) supplemented with $50 \mu \mathrm{g} / \mathrm{ml}$ RNase A. Eluates were acetone precipitated and the pellet was washed twice with $80 \%$ ethanol. The washed pellet was dissolved in $2 \times$ SDS sample by heating at $95^{\circ} \mathrm{C}$ for $5 \mathrm{~min}$. Western blot was performed to identify the RNA interacting proteins.

Triple-label SILAC-MS. Incorporation of Light (Lys0, Arg0), Medium (Lys4, Arg6), and Heavy (Lys8, Arg10) Isotopic Labels: The SILAC Protein Quantitation Kit RPMI-1640 (Life technologies, 8992) was used to generate light- and heavylabeled HLE cells. For generation of medium-labeled cells, 4,4,5,5-D4 L-Lysine$2 \mathrm{HCl}$ (Fischer Scientific, 11305402) and L-Arginine- $\mathrm{HCl}{ }^{13} \mathrm{C}_{6}$ (Life technologies, 88210 ) were individually purchased. 4,4,5,5-D4 L- Lysine- $2 \mathrm{HCl}$ and L-Arginine$\mathrm{HCl}{ }^{13} \mathrm{C}_{6}$ were mixed to achieve a final concentration of $0.46 \mathrm{mM}$ and $0.47 \mathrm{mM}$ respectively, in SILAC RPMI-1640 medium (Life Technologies, A2494401). Cells were grown in the respective medium supplemented with $200 \mu \mathrm{g} / \mathrm{ml} \mathrm{L}$-Proline (Thermo Fischer Scientific, 88211) for at least 8-10 passages in a humidified incubator at $37^{\circ} \mathrm{C}$ with $5 \% \mathrm{CO}_{2}$ to achieve $>99 \%$ incorporation of the respective labels as verified by mass spectrometry.

Cell culture, transfections, lysate preparation, protein quantification, LC-MS/ MS: After confirmation of label incorporation, isotopically labeled HLE cells were grown to $80 \%$ confluency. Cells were reverse transfected with the respective siPOOLs targeting lincNMR or the control siPOOL. Lysates were harvested $48 \mathrm{~h}$ post transfection. Cells were lysed in $200 \mu \mathrm{l}$ RIPA buffer $(50 \mathrm{mM}$ Tris- $\mathrm{HCl} \mathrm{pH} 7.5$, $150 \mathrm{mM} \mathrm{NaCl}, 1 \%$ Triton X-100, 0.5\% Na-deoxycholate, 0.1\% SDS, $1 \%$ DTT) supplemented with protease and phosphatase inhibitors. In addition, $0.1 \%$ benzonase (Merck, Darmstadt, Germany) was added to digest nucleic acids. After incubation on ice for $1 \mathrm{~h}$, cell lysates were cleared by centrifuging at $15,000 \mathrm{~g}$, for 30 min at $4^{\circ} \mathrm{C}$. The $2 \mathrm{D}$ Quant Kit (GE Healthcare, 806483356) was used to measure protein concentrations.

YBX1 ChIP assay. Chromatin immunoprecipitation (ChIP) assay was performed as described before ${ }^{74}$ to identify YBX1 interacting promoter regions. HLE cell lysates were used for the ChIP experiment using YBX1 antibody (Abcam, ab12148) and isotype control rabbit IgG antibody (Abcam, ab171870). ChIP pull-down DNA was analyzed using the qPCR and primers listed in Supplementary Data 8.

Dual-luciferase reporter assay. HLE and FLC-4 cells were reverse transfected with $10 \mathrm{nM}$ of control siPOOL and siPOOLs targeting lincNMR on day 1. Control pRL-TK/pRL-SV40 reporter constructs and Y-Box-TATA-Luc were co-transfected using a forward transfection protocol on day 2. Cell culture medium was changed on day 3. Luciferase reporter assay was performed using a Dual-Luciferase Reporter Assay System kit (Promega, E1960) on day 4 as per the manufacturer's instruction, and luminescence was measured on Spectra Max M5e (Molecular Devices).

For the characterization of the YBX1 impact on RRM2, TK1, and TYMS promoter regions, genomic DNA from HLE cells was PCR-amplified using Phusion polymerase (NEB, M0530). The first round of PCR was performed using primers without restriction sites. After gel elution of PCR purified products, a nested PCR was performed using primers with restriction sites. Gel purified PCR products and empty pcDNA3.1 vector were digested using Xhol / NotI and ligation was performed using T4 DNA ligase (Thermo, EL0011). Primers used for creating the constructs are listed in Supplementary Data 9.

dNTP quantification. Cellular dNTPs were extracted from cells (HLE and FLC-4) transfected with the respective siPOOLs $72 \mathrm{~h}$ post transfection as per a previously published protocol ${ }^{75}$. The dried dNTPs were resuspended to proper volumes of water and added to the HIV-1 RT-mediated single dNTP incorporation reactions. The percent of primer extension were converted to the incorporated dNTP amounts, and the determined dNTP amounts were normalized by 1 million cells for comparison. Further, data were normalized to negative control siPOOLs.

dNTP bathing and rescue assay. In total, 1250 cells were reverse transfected with the respective siPOOLs in a clear bottom 96 -well plate. The desired concentration of extracellular dNTPs was added to the cells $24 \mathrm{~h}$ later. A cell proliferation assay was performed at $96 \mathrm{~h}$ post siPOOL transfection to assess the proliferation of the cells.

RNA copy number detection. $1 \mu \mathrm{g}$ of RNA was used for reverse transcription with MaximaRT (Thermo Fischer EP0751) from respective cell lines (HLE, HLF, SNU387 and FLC-4). 1:10 diluted cDNA was used for qPCR. Primers used for qPCR are listed in Supplementary Data 4. Plasmids containing lincNMR-001 sequence were linearized using BstBI digestion and gel purified. Serial dilutions were made in the range of $0.02 \mathrm{pg}-5 \mathrm{pg}$. The copy number was calculated using the online tool https://cels.uri.edu/gsc/cndna.html.

Statistics and reproducibility. All experiments were performed at least in three biological replicates, and information about statistical tests used is detailed in the respective figure legends. Exact $P$-values are provided in the Source Data file. Numbers of replicates always refer to independent biological replicates.

Reporting summary. Further information on research design is available in the Nature Research Reporting Summary linked to this article.

\section{Data availability}

Sequencing data from $5^{\prime}$ - and $3^{\prime}$-RACE experiment supporting both lincNMR isoform have been deposited at Genbank with accession numbers MK652436 and MK652437. Source data for figures shown in this study are available upon request if not available in Supplementary Data and in the attached source data files. The source data underlying Fig. 4c, e and Supplementary Figs. 4e, f, 2i as well as Figs. 1a-d, f, g, 2a, b, d, 3c-g, 4d, f, 5a, b, d, f, 6d, e, 7a-d, f, and Supplementary Figs. 1e, f, 2a-h, 3a-d, 4c, g, h, 5b-e, 6a-c are provided as Source Data files.

Received: 19 April 2019; Accepted: 5 June 2020; Published online: 25 June 2020

\section{References}

1. Djebali, S. et al. Landscape of transcription in human cells. Nature $\mathbf{4 8 9}, 101-8$ (2012).

2. Batista, P. J. \& Chang, H. Y. Long noncoding RNAs: cellular address codes in development and disease. Cell 152, 1298-307 (2013).

3. Klingenberg, $\mathrm{M}$. et al. Non-coding RNA in hepatocellular carcinoma: mechanisms, biomarkers and therapeutic targets. J. Hepatol. 67, 603-618 (2017).

4. Hanahan, D. \& Weinberg, R. A. The hallmarks of cancer. Cell 100, 57-70 (2000)

5. Hanahan, D. \& Weinberg, R. A. Hallmarks of cancer: the next generation. Cell 144, 646-74 (2011)

6. Gutschner, T. \& Diederichs, S. The hallmarks of cancer: a long non-coding RNA point of view. RNA Biol. 9, 703-19 (2012).

7. $\mathrm{Li}, \mathrm{M}$. et al. An apela RNA-containing negative feedback loop regulates p53mediated apoptosis in embryonic stem cells. Cell Stem Cell 16, 669-83 (2015)

8. Mourtada-Maarabouni, M. et al. GAS5, a non-protein-coding RNA, controls apoptosis and is downregulated in breast cancer. Oncogene 28, 195-208 (2009)

9. Puvvula, P. K. et al. Long noncoding RNA PANDA and scaffold-attachmentfactor SAFA control senescence entry and exit. Nat. Commun. 5, 5323 (2014).

10. Takayama, K. et al. Androgen-responsive long noncoding RNA CTBP1-AS promotes prostate cancer. EMBO J. 32, 1665-80 (2013).

11. Tseng, Y. Y. et al. PVT1 dependence in cancer with MYC copy-number increase. Nature 512, 82-6 (2014).

12. Rinn, J. L. et al. Functional demarcation of active and silent chromatin domains in human HOX loci by noncoding RNAs. Cell 129, 1311-23 (2007).

13. Montes, M. et al. The lncRNA MIR31HG regulates p16(INK4A) expression to modulate senescence. Nat. Commun. 6, 6967 (2015).

14. Yoon, J. H. et al. LincRNA-p21 suppresses target mRNA translation. Mol. Cell 47, 648-55 (2012).

15. Liu, B. et al. A cytoplasmic NF-kappaB interacting long noncoding RNA blocks IkappaB phosphorylation and suppresses breast cancer metastasis. Cancer Cell 27, 370-81 (2015).

16. Flockhart, R. J. et al. BRAFV600E remodels the melanocyte transcriptome and induces BANCR to regulate melanoma cell migration. Genome Res. 22, 1006-14 (2012).

17. Gutschner, T. et al. The noncoding RNA MALAT1 is a critical regulator of the metastasis phenotype of lung cancer cells. Cancer Res. 73, 1180-9 (2013). 
18. Lu, Z. et al. Long non-coding RNA HULC promotes tumor angiogenesis in liver cancer by up-regulating sphingosine kinase 1 (SPHK1). Oncotarget 7 , 241-54 (2016).

19. Ji, P. et al. MALAT-1, a novel noncoding RNA, and thymosin beta4 predict metastasis and survival in early-stage non-small cell lung cancer. Oncogene 22, 8031-41 (2003).

20. Pandey, G. K. et al. The risk-associated long noncoding RNA NBAT-1 controls neuroblastoma progression by regulating cell proliferation and neuronal differentiation. Cancer Cell 26, 722-37 (2014).

21. Flynn, R. L. et al. TERRA and hnRNPA1 orchestrate an RPA-to-POT1 switch on telomeric single-stranded DNA. Nature 471, 532-6 (2011).

22. Kretz, M. et al. Control of somatic tissue differentiation by the long noncoding RNA TINCR. Nature 493, 231-5 (2013).

23. Yan, X. et al. Comprehensive genomic characterization of long non-coding RNAs across human cancers. Cancer Cell 28, 529-540 (2015).

24. Arun, G., Diermeier, S. D. \& Spector, D. L. Therapeutic targeting of long noncoding RNAs in cancer. Trends Mol. Med. 24, 257-277 (2018).

25. Wang, Y. et al. The long noncoding RNA IncTCF7 promotes self-renewal of human liver cancer stem cells through activation of Wnt signaling. Cell Stem. Cell 16, 413-25 (2015)

26. Chen, Z. Z. et al. LncSox4 promotes the self-renewal of liver tumour-initiating cells through Stat3-mediated Sox4 expression. Nat. Commun. 7, 12598 (2016).

27. Yuan, J. H. et al. A long noncoding RNA activated by TGF-beta promotes the invasion-metastasis cascade in hepatocellular carcinoma. Cancer Cell 25, 666-81 (2014)

28. Wang, J. et al. CREB up-regulates long non-coding RNA, HULC expression through interaction with microRNA-372 in liver cancer. Nucleic Acids Res. 38, 5366-83 (2010).

29. Bray, F. et al. Global cancer statistics 2018: GLOBOCAN estimates of incidence and mortality worldwide for 36 cancers in 185 countries. CA Cancer J. Clin. 68, 394-424 (2018).

30. Mitchell, M. et al. Structural basis for telomerase catalytic subunit TERT binding to RNA template and telomeric DNA. Nat. Struct. Mol. Biol. 17, 513-8 (2010).

31. Gupta, R. A. et al. Long non-coding RNA HOTAIR reprograms chromatin state to promote cancer metastasis. Nature 464, 1071-6 (2010).

32. Li, J. et al. TANRIC: an interactive open platform to explore the function of lncRNAs in cancer. Cancer Res. 75, 3728-37 (2015).

33. Hannus, M. et al. siPools: highly complex but accurately defined siRNA pools eliminate off-target effects. Nucleic Acids Res. 42, 8049-61 (2014).

34. Valianou, M. et al. Pharmacological inhibition of Polo-like kinase 1 (PLK1) by BI-2536 decreases the viability and survival of hamartin and tuberin deficient cells via induction of apoptosis and attenuation of autophagy. Cell Cycle 14, 399-407 (2015).

35. Yin, D. et al. Pro-angiogenic role of LncRNA HULC in microvascular endothelial cells via sequestrating miR-124. Cell Physiol. Biochem. 50, 2188-2202 (2018).

36. Parkhomchuk, D. et al. Transcriptome analysis by strand-specific sequencing of complementary DNA. Nucleic Acids Res. 37, e123 (2009).

37. Lin, M. F., Jungreis, I. \& Kellis, M. PhyloCSF: a comparative genomics method to distinguish protein coding and non-coding regions. Bioinformatics 27, i275-82 (2011).

38. Kong, L. et al. CPC: assess the protein-coding potential of transcripts using sequence features and support vector machine. Nucleic Acids Res. 35, W345-9 (2007).

39. Zhang, K. et al. The ways of action of long non-coding RNAs in cytoplasm and nucleus. Gene 547, 1-9 (2014).

40. Gandhi, M., Caudron-Herger, M. \& Diederichs, S. RNA motifs and combinatorial prediction of interactions, stability and localization of noncoding RNAs. Nat. Struct. Mol. Biol. 25, 1070-1076 (2018).

41. Munschauer, M. et al. The NORAD IncRNA assembles a topoisomerase complex critical for genome stability. Nature 561, 132-136 (2018).

42. Roth, A. \& Diederichs, S. Molecular biology: rap and chirp about X inactivation. Nature 521, 170-1 (2015).

43. Paz, I. et al. RBPmap: a web server for mapping binding sites of RNA-binding proteins. Nucleic Acids Res. 42, W361-7 (2014).

44. Marine, S. et al. Common seed analysis to identify off-target effects in siRNA screens. J. Biomol. Screen 17, 370-8 (2012).

45. Zhou, B. et al. A small-molecule blocking ribonucleotide reductase holoenzyme formation inhibits cancer cell growth and overcomes drug resistance. Cancer Res. 73, 6484-93 (2013).

46. Salama, R. et al. Cellular senescence and its effector programs. Genes Dev. 28, 99-114 (2014)

47. Blakley, R. L. \& Vitols, E. The control of nucleotide biosynthesis. Annu Rev. Biochem. 37, 201-24 (1968).

48. Nordlund, P. \& Reichard, P. Ribonucleotide reductases. Аnnu Rev. Biochem. 75, 681-706 (2006).
49. Reichard, P. Interactions between deoxyribonucleotide and DNA synthesis. Annu Rev. Biochem. 57, 349-74 (1988).

50. Aird, K. M. \& Zhang, R. Nucleotide metabolism, oncogene-induced senescence and cancer. Cancer Lett. 356, 204-10 (2015)

51. Aird, K. M. et al. Suppression of nucleotide metabolism underlies the establishment and maintenance of oncogene-induced senescence. Cell Rep. 3, 1252-65 (2013).

52. Mannava, S. et al. Depletion of deoxyribonucleotide pools is an endogenous source of DNA damage in cells undergoing oncogene-induced senescence. Am. J. Pathol. 182, 142-51 (2013).

53. Eriksson, S. \& Thelander, L. Allosteric regulation of calf thymus ribonucleotide reductase. Ciba Found. Symp. 68, 165-75 (1978)

54. Mannava, S. et al. Ribonucleotide reductase and thymidylate synthase or exogenous deoxyribonucleosides reduce DNA damage and senescence caused by C-MYC depletion. Aging 4, 917-22 (2012).

55. Zhao, X., Liu, Y. \& Yu, S. Long noncoding RNA AWPPH promotes hepatocellular carcinoma progression through YBX1 and serves as a prognostic biomarker. Biochim. Biophys. Acta Mol. Basis Dis. 1863, 1805-1816 (2017).

56. Liu, Y. et al. lncRNA GAS5 enhances G1 cell cycle arrest via binding to YBX1 to regulate p21 expression in stomach cancer. Sci. Rep. 5, 10159 (2015).

57. Peng, Z. et al. The long noncoding RNA LINC00312 induces lung adenocarcinoma migration and vasculogenic mimicry through directly binding YBX1. Mol. Cancer 17, 167 (2018).

58. Zhang, E. et al. A novel long noncoding RNA HOXC-AS3 mediates tumorigenesis of gastric cancer by binding to YBX1. Genome Biol. 19, 154 (2018).

59. Kosnopfel, C. et al. YB-1 expression and phosphorylation regulate tumorigenicity and invasiveness in melanoma by influencing EMT. Mol. Cancer Res. 16, 1149-1160 (2018).

60. Shibata, T. et al. Y-box binding protein YBX1 and its correlated genes as biomarkers for poor outcomes in patients with breast cancer. Oncotarget 9 , 37216-37228 (2018).

61. Seiler, J. et al. The IncRNA VELUCT strongly regulates viability of lung cancer cells despite its extremely low abundance. Nucleic Acids Res. 45, 5458-5469 (2017).

62. Manegold, C. et al. Gemcitabine in non-small cell lung cancer (NSCLC) Invest. N. Drugs 18, 29-42 (2000).

63. Aye, Y. \& Stubbe, J. Clofarabine $5^{\prime}$-di and -triphosphates inhibit human ribonucleotide reductase by altering the quaternary structure of its large subunit. Proc. Natl Acad. Sci. USA 108, 9815-20 (2011).

64. Aye, Y. et al. Clofarabine targets the large subunit (alpha) of human ribonucleotide reductase in live cells by assembly into persistent hexamers. Chem. Biol. 19, 799-805 (2012).

65. Pfisterer, J. et al. Combination therapy with gemcitabine and carboplatin in recurrent ovarian cancer. Int. J. Gynecol. Cancer 15, 36-41 (2005).

66. Levin, V. A. The place of hydroxyurea in the treatment of primary brain tumors. Semin Oncol. 19, 34-9 (1992).

67. Hehlmann, R. et al. Randomized comparison of busulfan and hydroxyurea in chronic myelogenous leukemia: prolongation of survival by hydroxyurea. The German CML Study Group. Blood 82, 398-407 (1993).

68. Sterkers, Y. et al. Acute myeloid leukemia and myelodysplastic syndromes following essential thrombocythemia treated with hydroxyurea: high proportion of cases with 17p deletion. Blood 91, 616-22 (1998).

69. Castro, F. et al. High-throughput SNP-based authentication of human cell lines. Int J. Cancer 132, 308-14 (2013).

70. Roth, A. et al. Targeting LINC00673 expression triggers cellular senescence in lung cancer. RNA Biol. 15, 1499-1511 (2018).

71. Gagnon, K. T. et al. Analysis of nuclear RNA interference in human cells by subcellular fractionation and Argonaute loading. Nat. Protoc. 9, 2045-60 (2014).

72. Franken, N. A. et al. Clonogenic assay of cells in vitro. Nat. Protoc. 1, 2315-9 (2006).

73. Hammerle, M. et al. Posttranscriptional destabilization of the liver-specific long noncoding RNA HULC by the IGF2 mRNA-binding protein 1 (IGF2BP1). Hepatology 58, 1703-12 (2013).

74. Caudron-Herger, M. et al. R-DeeP: proteome-wide and quantitative identification of RNA-dependent proteins by density gradient ultracentrifugation. Mol. Cell 75, 184-199 e10 (2019).

75. Diamond, T. L. et al. Macrophage tropism of HIV-1 depends on efficient cellular dNTP utilization by reverse transcriptase. J. Biol. Chem. 279, 51545-53 (2004).

\section{Acknowledgements}

We would like to thank PD Dr. Kai Breuhahn for providing HCC cell lines. We thank Dr. Monika Langlotz (FACS Facility, ZMBH Heidelberg) for sample acquisition 
of flow-cytometry experiments. We acknowledge Dr. Uwe Warnken and Dr. Martina Schnölzer for running the triple-label SILAC-MS at Proteomics Core Facility, DKFZ. We would like to thank Prof. Dr. Birgit Schittek and Dr. Corinna Kosnopfel for kindly sending the YB-1 reporter plasmid. We appreciate technical advice from Dr. Maria Polycarpou-Schwarz (SA- $\beta$ GAL Assay) and Dr. Marcel Klingenberg (CAM Assay). We thank Ramona Hempel, Jeannette Seiler, and Paula Eiben for technical assistance. This project was funded by the Deutsche Forschungsgemeinschaft to S.D., DFG Di 1421/7-1. M. Ga. was supported by a full doctoral scholarship from Deutscher Akademische Austauschdienst (DAAD). Research in the B.K. lab is supported by NIH GM104198, R01 AI136581, and R01 AI150451 grants.

\section{Author contributions}

M.Ga. designed and performed experiments, analyzed and interpreted the data. M.Gr., J.M.H., S.A.C., N.P., J.H.L., M.M., M.S., and C.H. performed experiments, and H.A. and B.K. supervised experiments. S.D. conceived and supervised the study. M.Ga. and S.D. wrote the paper. Parts of this study are part of M.Ga.'s doctoral thesis.

\section{Competing interests}

S.D. is co-owner of siTOOLs Biotech GmbH, Martinsried, Germany. The remaining authors declare no competing interests.

\section{Additional information}

Supplementary information is available for this paper at https://doi.org/10.1038/s41467020-17007-9.
Correspondence and requests for materials should be addressed to S.D.

Peer review information Nature Communications thanks the anonymous reviewer(s) for their contribution to the peer review of this work.

Reprints and permission information is available at http://www.nature.com/reprints

Publisher's note Springer Nature remains neutral with regard to jurisdictional claims in published maps and institutional affiliations.

(c) (i) Open Access This article is licensed under a Creative Commons Attribution 4.0 International License, which permits use, sharing, adaptation, distribution and reproduction in any medium or format, as long as you give appropriate credit to the original author(s) and the source, provide a link to the Creative Commons license, and indicate if changes were made. The images or other third party material in this article are included in the article's Creative Commons license, unless indicated otherwise in a credit line to the material. If material is not included in the article's Creative Commons license and your intended use is not permitted by statutory regulation or exceeds the permitted use, you will need to obtain permission directly from the copyright holder. To view a copy of this license, visit http://creativecommons.org/ licenses/by/4.0/.

(C) The Author(s) 2020 